\title{
Double Quantum Dot Photoluminescence Mediated by Incoherent Reversible Energy Transport
}

\author{
S. Yu Kruchinin \\ St. Petersburgh State University of Information Technologies, Mechanics and Optics
}

A. V. Federov

St. Petersburgh State University of Information Technologies, Mechanics and Optics

A. V. Baranov

St. Petersburgh State University of Information Technologies, Mechanics and Optics

See next page for additional authors

Follow this and additional works at: https://arrow.tudublin.ie/engscheceart

Part of the Electrical and Electronics Commons

\section{Recommended Citation}

Kruchinin, S. et al. (2010) Double Quantum Dot Photoluminescence Mediated by Incoherent Reversible Energy Transport. Physical Review B., Vol.81, no. 24, DOI:10.1103/PhysRevB.81.245303

This Article is brought to you for free and open access by the School of Electrical and Electronic Engineering at ARROW@TU Dublin. It has been accepted for inclusion in Articles by an authorized administrator of ARROW@TU Dublin. For more information, please contact arrow.admin@tudublin.ie, aisling.coyne@tudublin.ie, gerard.connolly@tudublin.ie.

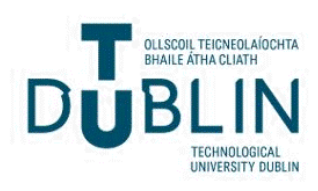




\section{Authors}

S. Yu Kruchinin, A. V. Federov, A. V. Baranov, T. S. Perova, and Kevin Berwick 


\title{
Double quantum dot photoluminescence mediated by incoherent reversible energy transport
}

\author{
S. Yu. Kruchinin, ${ }^{*}$ A. V. Fedorov ${ }^{\dagger}$ and A. V. Baranov \\ Saint-Petersburg State University of Information Technologies, Mechanics and Optics, \\ 49 Kronverksky Avenue, 197101 St. Petersburg, Russia \\ T. S. Perova \\ Department of Electronic and Electrical Engineering, Trinity College, University of Dublin, Dublin 2, Ireland \\ K. Berwick \\ Department of Electronic and Communications Engineering, Dublin Institute of Technology, Dublin 8, Ireland
}

(Received 25 March 2010; published 2 June 2010)

\begin{abstract}
We present a theoretical study of the stationary photoluminescence of two, direct-gap, semiconductor nanocrystals, taking into account electronic excitation energy-transfer processes due to electrostatic interaction. The results obtained here allow for the incoherent reversible energy transport that occurs when the intraband relaxation rate in a quantum dot acceptor is comparable to, or less than, the energy-transfer rate. We investigate the secondary emission of two different electronic level schemes that can be realized experimentally, obtain analytical expressions for the luminescence differential cross section, and perform an analysis of its spectrum. It is shown that when excitation is not in resonance with the levels involved in energy transfer, the energy transfer is more efficient.
\end{abstract}

DOI: 10.1103/PhysRevB.81.245303

\section{INTRODUCTION}

A fundamental problem in low-dimensional physics is the energy transport of elementary excitations in nanostructures and, in particular, the nonradiative energy transfer between semiconductor quantum dots (QDs). The problem is especially important for structures with a close-packed arrangement of QDs, i.e., when the interdot distance is on the order of the QD size or smaller. Examples of these structures are quantum dot molecules, ${ }^{1}$ chains, ${ }^{2}$ rings,${ }^{3}$ two-dimensional ${ }^{4}$ and three-dimensional ${ }^{5}$ arrays as well as dendrites ${ }^{6,7}$ based on QDs.

Over small interdot distances, the electrostatic interaction between QD electronic subsystems is an important factor that determines the electronic and optical properties of QD ensembles. This interaction can lead to both incoherent and coherent energy transfer between QDs, just as in atomic and molecular systems. ${ }^{8}$ The presence or absence of coherent effects in energy transfer from the QD donor to the QD acceptor is determined by the relationship between the transfer rate $\gamma_{D A}$ and the dephasing rate $\Gamma_{D A}$ for this transition. If $\Gamma_{D A}$ $\gg \gamma_{D A}$, then incoherent energy transfer takes place; otherwise coherent effects will influence the energy transport between QDs. It is also important to distinguish between the two regimes of incoherent energy transfer that differ from each other by the relationship between $\gamma_{D A}$ and the intraband relaxation rate $\gamma_{A}$ of the QD acceptor. Firstly, if $\gamma_{D A} \ll \gamma_{A}$, then all the electron-hole pairs excited in the QD acceptor due to energy transfer will quickly relax to the lowest energy state of the QD acceptor and the energy transport will be irreversible. Secondly, if $\gamma_{D A} \geqslant \gamma_{A}$, then some of the electron-hole pairs of the QD acceptor will return their energy back to the QD donor because of the energy-transfer process. So, reversible energy transport will take place in this case.

When the highly excited state of the QD acceptor is involved in resonant energy transfer, a significant contribution to $\gamma_{A}$ and $\Gamma_{D A}$ is provided by nonradiative intraband relax-
PACS number(s): 78.67.Hc, 78.55.-m, 73.21.La

ation, so the question arises as to whether it is possible to obtain incoherent reversible and coherent $\left(\gamma_{D A} \gg \Gamma_{D A}\right)$ energy transfer in actual QD systems. There are numerous experimental observations of fast picosecond and even femtosecond intraband relaxation in the literature. In particular, relaxation occurring over these time scales were observed for colloidal QDs. ${ }^{9-11}$ However, there is also evidence of slow intraband relaxation, on the order of tens of picoseconds ${ }^{12}$ or even nanoseconds, ${ }^{13}$ for similar QDs. Low relaxation rates have been reported for self-assembled QDs in Ref. 14 (InAs/ GaInP QDs) and in Ref. 15 ( $\operatorname{In}_{x} \mathrm{Ga}_{1-x} \mathrm{As} / \mathrm{GaAs}$ QDs). These findings show that the intraband carrier relaxation rate in QDs is subject to wide variations from $10^{8}$ to $10^{13} \mathrm{~s}^{-1}$, and depends on the growth process, size, shape, QD material, and the properties of the environment.

To allow interpretation of experimental results, various mechanisms for intraband carrier relaxation have been proposed. A reduction in the intraband relaxation rates in QDs as compared with bulk materials has been predicted in Refs. 16-18. Fast intraband carrier relaxation can be explained by multiphonon processes involving point defects, ${ }^{19-21}$ Augertype processes, ${ }^{22-24}$ processes mediated by plasmon and plasmon-LO-phonon emission, ${ }^{25-27}$ as well as transitions via surface ligand states. ${ }^{12,28}$ Obviously, the dominant relaxation mechanisms will depend on a number of parameters, including QD size and shape, QD and matrix materials, and specimen temperature. Since the energy transfer rate $\gamma_{D A}$ can reach values on the order of $10^{12} \mathrm{~s}^{-1},{ }^{29}$ one should expect that incoherent reversible and coherent energy transfer would exist in real QD systems, and therefore a study of these processes is an important problem.

Resonant energy transfer should be apparent in the optical spectra of interacting QDs and, in particular, in their luminescence spectra. The effect has been observed in photoluminescence experiments in the frequency domain ${ }^{30-34}$ and time domain. ${ }^{30,35-38}$ Although many theoretical studies have been devoted to the investigation of a variety of aspects of 
energy transport in QD systems, ${ }^{39-42}$ a consistent theoretical description of secondary emission for these systems, taking into account energy transfer, has not been proposed.

In Ref. 29 the theory of stationary photoluminescence for double quantum dots with incoherent irreversible energy transfer has been developed. However, two other regimes, namely, incoherent reversible and coherent energy transport were not considered in this study. So, development of a theory of double quantum dot photoluminescence for these regimes is of importance.

This study is devoted to an investigation of double quantum dot stationary photoluminescence, taking into account incoherent reversible energy transport between QDs. Two distinct types of transitions between QD electronic states have been considered. These two schemes are sufficient to describe photoluminescence processes for double QDs. Analytical expressions for the photoluminescence cross sections of QD donors and QD acceptors as a function of the interdot distance and QD relaxation parameters have been derived. A comparison of the results obtained with calculations carried out for incoherent irreversible energy transfer ${ }^{29}$ has been performed.

\section{GENERAL MODEL}

Let us consider the dynamics of quantum transitions for double quantum dots, consisting of a QD donor and a QD acceptor, whose electronic subsystems interact with a classical optical field and the quantum electromagnetic field of the vacuum. In addition, we assume that the QDs are coupled to each other via a screened Coulomb potential.

The Hamiltonian of the two uncoupled QDs can be represented in the following form:

$$
H_{0}=H_{D}+H_{A}+H_{R}+H_{D R}+H_{D L}+H_{A R}+H_{A L},
$$

where

$$
H_{\alpha}=\sum_{i} \hbar \omega_{i \alpha}|i \alpha\rangle\langle i \alpha|
$$

are the Hamiltonians of noninteracting electron-hole pairs in the QD donor $(\alpha=D)$ and QD acceptor $(\alpha=A)$ in terms of their eigenvectors $|i \alpha\rangle$ and eigenvalues $\hbar \omega_{i \alpha}$,

$$
H_{R}=\sum_{k} \hbar \omega_{k} b_{k}^{+} b_{k}
$$

is the Hamiltonian of the electromagnetic field, $b_{k}^{+}$and $b_{k}$ are the operators of the creation and annihilation of photons of the $k$ mode with frequency $\omega_{k}$,

$$
\begin{gathered}
H_{\alpha R}=\sum_{i, k} g_{\alpha k}\left(i \hbar V_{i \alpha, 0 \alpha}^{(k)} b_{k}|i \alpha\rangle\langle 0 \alpha|+\text { H.c. }\right), \\
H_{\alpha L}=\sum_{i}\left[\phi(t) V_{i \alpha, 0 \alpha}^{(L)} e^{-i \omega_{L} t}|i \alpha\rangle\langle 0 \alpha|+\text { H.c. }\right]
\end{gathered}
$$

are the operators describing the interaction of the electronhole pairs of the QD donor and QD acceptor with the quantum electromagnetic field and the classical optical field with frequency $\omega_{L},|0 \alpha\rangle$ is the vacuum state of the electron-hole pairs, $g_{\alpha k}=\sqrt{2 \pi \omega_{k} / \varepsilon_{\alpha} \hbar V}, \varepsilon_{\alpha}$ is the dielectric constant of the corresponding QD, $V$ is the normalization volume, $V_{i \alpha, 0 \alpha}^{(\eta)}$ $=\left\langle i \alpha\left|(-e \mathbf{r}) \mathbf{e}_{\eta}\right| 0 \alpha\right\rangle, \eta=L, k, e \mathbf{r}$ is the dipole moment operator, $\mathbf{e}_{\eta}$ are the polarization vectors of the photon, $\phi(t)$ is the complex time-dependent amplitude of the classical optical field.

The dynamics of the quantum transitions of uncoupled QDs under the influence of the quantum electromagnetic field and the classical optical field may be described by the generalized master equation for the reduced density matrix,

$$
\dot{\rho}_{i \alpha, j \alpha}=\frac{1}{i \hbar}[H, \rho]_{i \alpha, j \alpha}+\delta_{i \alpha, j \alpha} \sum_{k \neq j} \zeta_{j \alpha, k \alpha} \rho_{k \alpha, k \alpha}-\gamma_{i \alpha, j \alpha}^{(0)} \rho_{i \alpha, j \alpha},
$$

where $\gamma_{i \alpha, i \alpha}^{(0)}$ is the population relaxation rate of state $i$, which is inversely proportional to its lifetime, $\gamma_{i \alpha, j \alpha}^{(0)}=\left(\gamma_{i \alpha, j \alpha}^{(0)}\right.$ $\left.+\gamma_{j \alpha, j \alpha}^{(0)}\right) / 2+\bar{\gamma}_{i \alpha, j \alpha}^{(0)}$ for $i \neq j$ is the dephasing rate of the transition $|j \alpha\rangle \rightarrow|i \alpha\rangle, \bar{\gamma}_{i \alpha, j \alpha}^{(0)}=\bar{\gamma}_{j \alpha, i \alpha}^{(0)}$ is the pure dephasing rate of the corresponding transition, $\zeta_{i \alpha, j \alpha}$ is the rate of transition $|j \alpha\rangle \rightarrow|i \alpha\rangle$ due to interaction with the bath. This approach implies that the bath is weakly coupled to the dynamic system and possesses only a short-term memory. ${ }^{43}$ So, according to Fano, ${ }^{44}$ the relaxation superoperator is reduced to a set of constants that determine the dynamics of the diagonal and nondiagonal elements of the reduced density matrix. We also assume that these constants are real, i.e., the shift of the energy levels due to interaction with the bath is ignored.

Coulomb interaction between the QD electronic subsystems in the incoherent reversible regime can be taken into account by a semiphenomenological approach based on the energy transfer rate $\gamma_{D A}$, just as has been done for the incoherent irreversible regime. ${ }^{29}$ In this case, additional terms due to the creation and annihilation of electron-hole pairs as a result of interdot Coulomb interaction arise in Eq. (1), describing the QD donor and QD acceptor evolution. As a result, instead of independent subsystems of kinetic equations for the donor and acceptor, we obtain a system of coupled equations for the double quantum dot. Evidently, the additional terms arise only for the equations that connect the QD states involved in the energy-transfer process. For example, if energy transfer between the $i D$ state of the donor and the $j A$ state of the acceptor takes place at a rate $\gamma_{D A}$, the corresponding equations for the density matrix elements, Eq. (1), are modified and are given by

$$
\begin{aligned}
\dot{\rho}_{i D, i D}= & \frac{1}{i \hbar}[H, \rho]_{i D, i D}+\sum_{p \neq i} \zeta_{i D, p D} \rho_{p D, p D}-\gamma_{i D, i D} \rho_{i D, i D} \\
& +\gamma_{D A} \rho_{j A, j A}, \\
\dot{\rho}_{j A, j A}= & \frac{1}{i \hbar}[H, \rho]_{j A, j A}+\sum_{k \neq j} \zeta_{j A, k A} \rho_{k A, k A}-\gamma_{j A, j A} \rho_{j A, j A} \\
& +\gamma_{D A} \rho_{i D, i D}, \\
& \dot{\rho}_{i D, k D}=\frac{1}{i \hbar}[H, \rho]_{i D, k D}-\gamma_{i D, k D} \rho_{i D, k D},
\end{aligned}
$$




$$
\dot{\rho}_{j A, k A}=\frac{1}{i \hbar}[H, \rho]_{j A, k A}-\gamma_{j A, k A} \rho_{j A, k A},
$$

where

$$
\begin{gathered}
\gamma_{i D, i D(j A, j A)}=\gamma_{i D, i D(j A, j A)}^{(0)}+\gamma_{D A}, \\
\gamma_{i D, k D(j A, k A)}=\gamma_{i D, k D(j A, k A)}^{(0)}+\gamma_{D A} / 2 .
\end{gathered}
$$

For simplicity, we have neglected a possible degeneration of the $i D$ state of the donor and the $j A$ state of the acceptor in Eqs. (2)-(5).

Using this approach, the energy transfer rate is calculated quantum mechanically and is of the form

$$
\gamma_{D A}=\frac{2}{\hbar^{2}}\left|\left\langle 0 D, j A\left|V_{C}\right| i D, 0 A\right\rangle\right|^{2} \frac{\Gamma_{D A}}{\Gamma_{D A}^{2}+\Delta_{D A}^{2}},
$$

where $V_{C}$ is the screened interdot Coulomb potential,

$$
V_{C}\left(\mathbf{r}, \mathbf{r}_{D}, \mathbf{r}_{A}\right)=\frac{e^{2}}{\varepsilon\left|\mathbf{r}+\mathbf{r}_{D}-\mathbf{r}_{A}\right|},
$$

$\mathbf{r}_{D}$ and $\mathbf{r}_{A}$ are the radius vectors of electrons, originating from the center of the corresponding quantum dot, $\mathbf{r}$ is the vector directed from the center of the acceptor to the center of the donor, and $\varepsilon$ is the effective dielectric constant, ${ }^{41}$

$$
\varepsilon=\frac{\left(\varepsilon_{D}+2 \varepsilon_{M}\right)\left(\varepsilon_{A}+2 \varepsilon_{M}\right)}{9 \varepsilon_{M}}
$$

$\varepsilon_{D}, \varepsilon_{A}$, and $\varepsilon_{M}$ are the high-frequency dielectric constants of the donor, acceptor, and matrix, respectively,

$$
\Gamma_{D A}=\gamma_{i D, 0 D}^{(0)}+\gamma_{j A, 0 A}^{(0)}
$$

is the dephasing rate of the interdot transition with energy transfer, $\Delta_{D A}=\omega_{i D}-\omega_{j A}$ is the detuning between the interband transition frequencies of the QD donor and the QD acceptor.

We restrict our consideration to a strong confinement mode for the spherical QD donor and QD acceptor with radii $R_{D}$ and $R_{A}$, respectively. Furthermore, we assume that these QDs are embedded in a dielectric matrix, so we can use an infinite potential wall model. This approach adequately describes the electronic structure of spherical semiconductor QDs formed in organic and aqueous solutions by the hotinjection method, ${ }^{30,45}$ as well as in a glass matrix by diffusion phase decomposition of a supersaturated solid solution of the basic constituents under secondary heat treatment. ${ }^{46,47}$ Systems formed from such QDs demonstrate energy-transfer properties and offer considerable promise for biosensor and light harvesting applications. $., 30,48$

As before, ${ }^{29}$ we use the simple two-band approximation ( $c$ is the conduction band and $v$ is the valence band) to describe the QD states. In this case, the energies and wave functions of the QD electron-hole pairs are given by the following expressions:

$$
\hbar \omega_{i \alpha}=E_{g}^{(\alpha)}+E_{n_{\alpha} l^{m^{\prime}} m^{(c)}}^{(c)}+E_{n_{\alpha^{\prime} l^{\prime} m_{\alpha}^{\prime}}^{(v)}}=E_{g}^{(\alpha)}+\frac{\hbar^{2} \xi_{n_{\alpha} l_{\alpha}}^{2}}{2 m_{c}^{(\alpha)} R_{\alpha}^{2}}+\frac{\hbar^{2} \xi_{n_{\alpha}^{\prime} l^{\prime}}^{2}}{2 m_{v}^{(\alpha)} R_{\alpha}^{2}}
$$

and

$$
\begin{aligned}
|i \alpha\rangle= & \left|c, n_{\alpha}, l_{\alpha}, m_{\alpha}\right\rangle\left|v, n_{\alpha}^{\prime}, l_{\alpha}^{\prime}, m_{\alpha}^{\prime}\right\rangle \\
= & u_{\alpha c}\left(\mathbf{r}_{\alpha}\right) R_{n_{\alpha} l_{\alpha}}\left(r_{\alpha}\right) Y_{l_{\alpha}, m_{\alpha}}\left(\theta_{\alpha}, \phi_{\alpha}\right) \\
& \times u_{\alpha v}^{*}\left(\mathbf{r}_{\alpha}\right) R_{n_{\alpha}^{\prime} l^{\prime}}^{*}\left(r_{\alpha}\right) Y_{l^{\prime}, m_{\alpha}^{\prime}}^{*}\left(\theta_{\alpha}, \phi_{\alpha}\right)
\end{aligned}
$$

denote the state of the electron-hole pair, where

$$
R_{n_{\alpha} l_{\alpha}}\left(r_{\alpha}\right)=\sqrt{\frac{2}{R_{\alpha}^{3}}} \frac{j_{l_{\alpha}}\left(\xi_{n_{\alpha} l_{\alpha}} r_{\alpha} / R_{\alpha}\right)}{j_{l_{\alpha}+1}\left(\xi_{n_{\alpha} l_{\alpha}}\right)}
$$

is the radial part of the envelope function, $u_{\alpha c}\left(\mathbf{r}_{\alpha}\right)$ and $u_{\alpha v}\left(\mathbf{r}_{\alpha}\right)$ are the Bloch amplitudes, $Y_{l_{\alpha}, m_{\alpha}}$ is the spherical harmonic, $j_{l}(x)$ is the spherical Bessel function, $\xi_{n l}$ is the $n$th root of equation $j_{l}(x)=0, E_{g}^{(\alpha)}$ is the semiconductor band gap, $m_{c}^{(\alpha)}$ and $m_{v}^{(\alpha)}$ are the electron and hole effective masses, respectively, $n, l$, and $m$ are the principal quantum number, the angular momentum, and its projection for electron or hole states. We will use the following notation for the states of the electron-hole pairs in donors $(\alpha=D)$ and acceptors $(\alpha=A): i \alpha \equiv\left\{c n_{2} l_{2} m_{2} ; v n_{1} l_{1} m_{1}\right\} \alpha$.

According to Ref. 29, the matrix element of the screened, interdot Coulomb potential for spherical QDs based on direct-gap semiconductors using a two-band approximation is given by

$$
M_{D A} \equiv\left\langle 0 D, j A\left|V_{C}\right| i D, 0 A\right\rangle=\frac{e^{2}}{\varepsilon r^{3}}\left|\mathbf{r}_{v c}^{(D)} \| \mathbf{r}_{c v}^{(A)}\right| \bar{\chi},
$$

where

$$
\bar{\chi}=I_{1} \sin \theta_{D} \sin \theta_{A} \cos \phi+\left(I_{1}-I_{2}\right) \cos \theta_{D} \cos \theta_{A},
$$

$\mathbf{r}_{v c}^{(\alpha)}$ is the matrix element of the coordinate operator between the Bloch functions, expressed using the material parameters of the bulk semiconductor as follows:

$$
\left|\mathbf{r}_{v c}^{(\alpha)}\right|=\frac{P^{(\alpha)}}{E_{g}^{(\alpha)}}
$$

Here $P^{(\alpha)}=\hbar^{2} / m_{0}\langle S|\partial / \partial z| Z\rangle$ is the Kane parameter ${ }^{49}$ and $m_{0}$ is the free-electron mass. We use a spherical coordinate system (Fig. 1) where the $z$ axis is parallel to $\mathbf{r}$, vectors $\mathbf{r}_{v c}^{(D)}$ and $\mathbf{r}_{c v}^{(A)}$ make angles $\theta_{D}$ and $\theta_{A}$ with $\mathbf{r}$, respectively, and $\phi$ is the difference between their azimuth angles. In Eq. (14),

$$
I_{l}=\frac{2}{\pi} \sum_{l_{1}=0}^{l_{D}^{+l_{D}^{\prime}}} \sum_{l_{2}=0}^{l_{A}+l_{A}^{\prime}} \mathfrak{C}_{D, l_{1}}^{(+)} \mathfrak{C}_{A, l_{2}}^{(-)} \int_{0}^{R_{D}} \int_{0}^{R_{A}} d r_{D} d r_{A} r_{D}^{2} r_{A}^{2} \mathfrak{R}_{D} \Re_{A} Q_{l_{1}, l_{2}}^{l}
$$

are the multipole amplitudes with

$$
\mathfrak{C}_{\alpha, k}^{( \pm)}=( \pm i)^{k}(2 k+1) \sqrt{\frac{2 l_{\alpha}+1}{2 l_{\alpha}^{\prime}+1}} C_{l_{\alpha}^{0, k 0}}^{l_{\alpha}^{\prime} 0} C_{l_{\alpha} m_{\alpha} l_{\alpha}^{m_{\alpha}^{\prime}},}^{l^{\prime}},
$$




$$
\begin{gathered}
Q_{l_{1}, l_{2}}^{l}=2^{l-3} \pi^{3 / 2}\left(\frac{r_{D}}{r}\right)^{l_{1}}\left(\frac{r_{A}}{r}\right)^{l_{2}} \Gamma\left[\begin{array}{c}
\left(2 \ell+l_{1}+l_{2}+1\right) / 2 \\
l_{1}+3 / 2, l_{2}+3 / 2,1-\left(l_{1}+l_{2}\right) / 2
\end{array}\right] \times F_{4}\left(\frac{l_{1}+l_{2}}{2}, \frac{2 l+l_{1}+l_{2}+1}{2} ; l_{1}+\frac{3}{2}, l_{2}+\frac{3}{2} ; \frac{r_{D}^{2}}{r^{2}} ; \frac{r_{A}^{2}}{r^{2}}\right) \\
\Gamma\left[\begin{array}{c}
a \\
b, c, d
\end{array}\right]=\frac{\Gamma(a)}{\Gamma(b) \Gamma(c) \Gamma(d)},
\end{gathered}
$$

$C_{l_{\alpha} m_{\alpha}}^{l_{\alpha}^{m_{\alpha}} m_{\alpha 0}^{\prime}}$ is the Clebsch-Gordan coefficient, $\Gamma(a)$ is the gamma function, $F_{4}\left(a, b ; c, c^{\prime} ; x ; y\right)$ is the Appel's fourth hypergeometric function,

$$
\Re_{\alpha}=R_{n_{\alpha}^{\prime} l^{\prime}}^{*}\left(r_{\alpha}\right) R_{n_{\alpha} l_{\alpha}}\left(r_{\alpha}\right)
$$

is the radial part of the electron-hole pair envelope wave function. Here, the unprimed symbols $n_{\alpha}, l_{\alpha}, m_{\alpha}$ indicate the initial states while the primed symbols $n_{\alpha}^{\prime}, l_{\alpha}^{\prime}, m_{\alpha}^{\prime}$ correspond to the final states of the donor and acceptor.

Assuming that the QDs are made of the same material, we can simplify Eq. (13),

$$
M_{D A}=\frac{\bar{\chi}}{\varepsilon r^{3}}\left(\frac{e P}{E_{g}}\right)^{2} .
$$

Function $\bar{\chi}$, defined by Eq. (14), is the generalization of the dipole-dipole orientation factor $\chi$ to the case of dipoleforbidden interband transitions. If both transitions in the QDs are dipole allowed, then $I_{1}=1, I_{2}=3$, and Eq. (14) transforms to the well-known expression, ${ }^{8}$

$$
\chi\left(\theta_{D}, \theta_{A}, \phi\right)=\sin \theta_{D} \sin \theta_{A} \cos \phi-2 \cos \theta_{D} \cos \theta_{A} .
$$

If at least one interband transition in the quantum dots is dipole forbidden, then $\bar{\chi}$ will depend on the quantum numbers of the corresponding QD states and radii, as well as on the interdot distance. Value of $|\bar{\chi}|^{2}$ change from 0 to $\mid I_{1}$ $-\left.I_{2}\right|^{2}$, depending on the interband dipole moment orientation. Below, we will consider a maximal value of the matrix element, Eq. (19), since the effects connected with changing the intraband relaxation and dephasing rates are most apparent

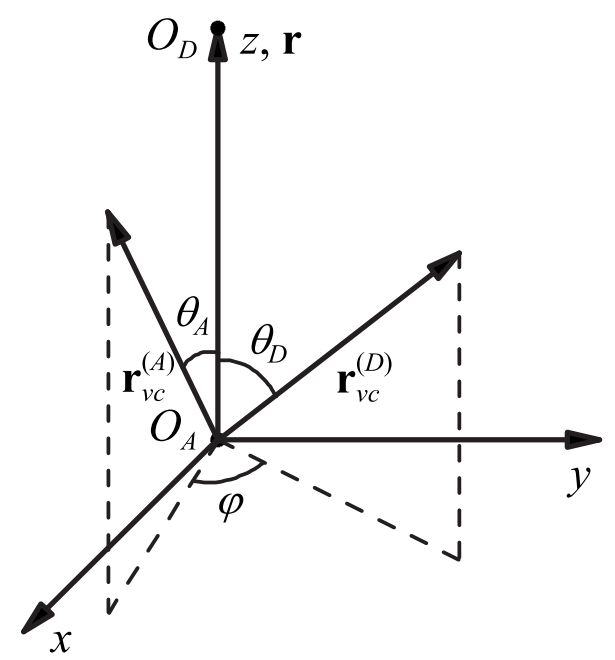

FIG. 1. Mutual orientation of vector $\mathbf{r}$ directed from the center of the acceptor to the center of the donor and vectors $\mathbf{r}_{v c}^{(D)}$ and $\mathbf{r}_{c v}^{(A)}$. under this condition. The conditions for $|\bar{\chi}|^{2}$ to be a maximum are (1) $\theta_{D}=0, \theta_{A}=0$; (2) $\theta_{D}=0, \theta_{A}=\pi$; (3) $\theta_{D}=\pi, \theta_{A}$ $=0$; and (4) $\theta_{D}=\pi, \theta_{A}=\pi$, and we obtain

$$
\max \left|M_{D A}\right|^{2}=\frac{\left|I_{1}-I_{2}\right|^{2}}{\varepsilon^{2} r^{6}}\left(\frac{e P}{E_{g}}\right)^{4} .
$$

In particular, if the transitions in the QD donor and QD acceptor are dipole allowed, then $\left|I_{1}-I_{2}\right|^{2}=4$.

In our numerical calculations, we consider quantum dots formed from the cubic modification of CdSe (Ref. 50): $m_{c}^{(\alpha)}=0.11 m_{0}, \quad m_{v}^{(\alpha)}=1.14 m_{0}, \quad E_{g}^{(\alpha)}=1.736 \mathrm{eV}, \quad P=1.48$ $\times 10^{-19} \mathrm{~cm}^{3} \mathrm{~g} \mathrm{~s}^{-2}, \varepsilon_{\alpha}=5.8$, embedded in a $\mathrm{SiO}_{2}$ matrix with a high-frequency dielectric constant $\varepsilon_{M}=2.13$. $^{51}$

Let us find the transitions that maximize the square modulus of the energy-transfer matrix element for this case. Figure 2 shows the dependencies of the square modulus of the matrix element on the distance between the quantum dot surfaces $R=r-R_{D}-R_{A}$ for several low-energy transitions. We do not consider the trivial case where the resonant energy transfer occurs between the fundamental transitions of the donor and acceptor and they have the same radii. Figure 2 shows that maximal values of the matrix element can be achieved for energy transfer to $\{c 110 ; v 110\} A$ state among dipoleallowed transitions and to $\{c 100 ; v 110\} A$ among dipoleforbidden transitions. A comparison of the matrix elements for different acceptor states allows us to make the following conclusions. The optimal case for observation of resonant energy transfer in nanocrystals of direct-band wide-gap
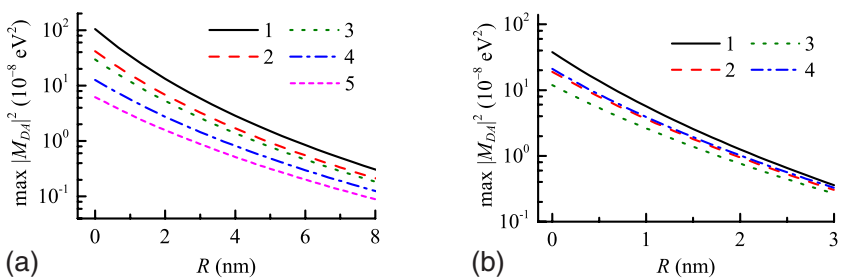

FIG. 2. (Color online) Dependencies of energy-transfer matrix element on intersurface distance $R=r-R_{D}-R_{A}$ for different interband transitions in a QD acceptor. In this calculation, we use a fixed donor radius $R_{D}=2 \mathrm{~nm}$ and consider resonant energy transfer from the lowest-energy state of the donor. Acceptor radii are obtained from the resonance condition $\omega_{i D}\left(R_{D}\right)=\omega_{j A}\left(R_{A}\right)$. (a) Dipole-allowed transitions. Numbers denote energy transfer with creation of the following electron-hole pairs in the acceptor: $1-c 110 ; v 110\left(R_{A}\right.$ $=2.86 \mathrm{~nm}), 2-c 120 ; v 120\left(R_{A}=3.67 \mathrm{~nm}\right), 3-c 200 ; v 200\left(R_{A}\right.$ $=4 \mathrm{~nm}), 4-c 210 ; v 210\left(R_{A}=4.92 \mathrm{~nm}\right)$, and 5-c220;v220 $\left(R_{A}\right.$ $=5.79 \mathrm{~nm})$. (b) Dipole-forbidden transitions: $1-c 100 ; v 110\left(R_{A}\right.$ $=2.09 \mathrm{~nm}), 2-c 110 ; v 100 \quad\left(R_{A}=2.80 \mathrm{~nm}\right), 3-c 120 ; v 110 \quad\left(R_{A}\right.$ $=2.94 \mathrm{~nm})$, and $4-c 110 ; v 120\left(R_{A}=3.61 \mathrm{~nm}\right)$. 


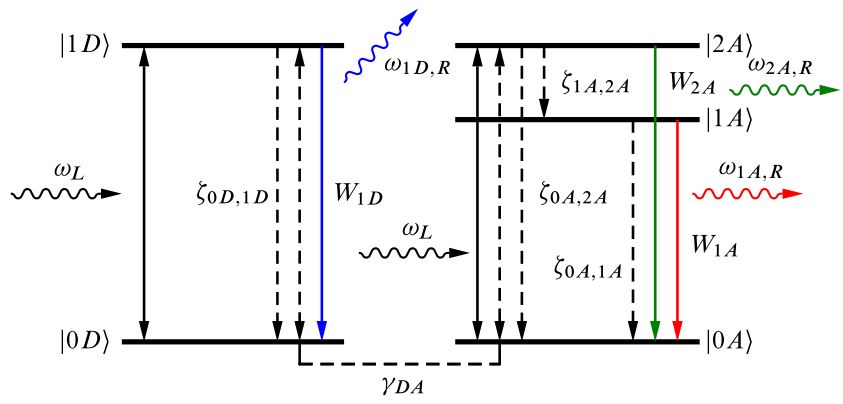

FIG. 3. (Color online) 2-3 scheme of double quantum dot for the allowed optical transitions in QD acceptor. $\zeta_{0 D, 1 D}, \zeta_{0 A, 1 A}, \zeta_{0 A, 2 A}$ are the interband relaxation rates for the QD donor and QD acceptor. $W_{1 D}, W_{1 A}, W_{2 A}$ are the spontaneous light emission rates for the QD donor and QD acceptor. $\zeta_{1 A, 2 A}$ is the intraband relaxation rate for the QD acceptor. $\gamma_{D A}$ is the energy-transfer rate. $\omega_{1 D, R}, \omega_{1 A, R}, \omega_{2 A, R}$ are the spontaneous light emission frequencies. $\omega_{L}$ is the incident light frequency. $|0 D\rangle,|0 A\rangle$ and $|1 D\rangle,|1 A\rangle$ are the ground and lowest-energy states of the QD donor and QD acceptor. $|2 A\rangle$ is the high-energy state of the QD acceptor.

semiconductors is realized when one of the high-energy acceptor states is in resonance with the lowest-energy donor state $\{c 100, v 100\} D$ because interband relaxation rates in these materials are smaller than the intraband rate. Due to the strong dependence of the matrix element on interdot distance (polynomial over $r$ ) the maximal efficiency of resonant energy transfer is achieved by quantum dots of the smallest possible radius, i.e., for the lowest-energy excited state in the acceptor. Therefore, in subsequent calculations we will use the transitions $\{c 100 ; v 100\} D \rightarrow\{c 110 ; v 110\} A$ and $\{c 100 ; v 100\} D \rightarrow\{c 100 ; v 110\} A$ as examples of dipoledipole and dipole-multipole resonant energy-transfer processes, respectively.

\section{FREQUENCY DOMAIN SPONTANEOUS LIGHT EMISSION}

Based on the model developed in the previous section, we can investigate frequency domain spontaneous light emission from double quantum dots. It is necessary to distinguish two cases that differ each other by the photoexcitation conditions. The first case occurs when incident light at a frequency $\omega_{L}$ creates electron-hole pairs in the lowest-energy state $|1 D\rangle$ of the QD donor (Figs. 3 and 4). In order to describe this process it is sufficient to use a so-called 2-3 scheme, i.e., a two-level model for the QD donor and a three-level model for the QD acceptor. Three relaxation channels for photoexcited electron-hole pairs are possible. The first channel involve the interband relaxation to the QD-donor ground state $|0 D\rangle$ at a rate $\zeta_{0 D, 1 D}$. The second channel involve the radiative recombination of the QD-donor electron-hole pairs at a rate $W_{1 D}$ and emission of photons at a frequency $\omega_{1 D, R}$. The third channel involve the nonradiative recombination of the QD-donor electron-hole pairs and transfer of their energy at a rate $\gamma_{D A}$ due to interdot Coulomb interaction between the QD donor and the QD acceptor creating electron-hole pairs in the high-energy state $|2 A\rangle$ of the QD acceptor, which is in resonance with the $|1 D\rangle$ state. Since the electron-hole pair

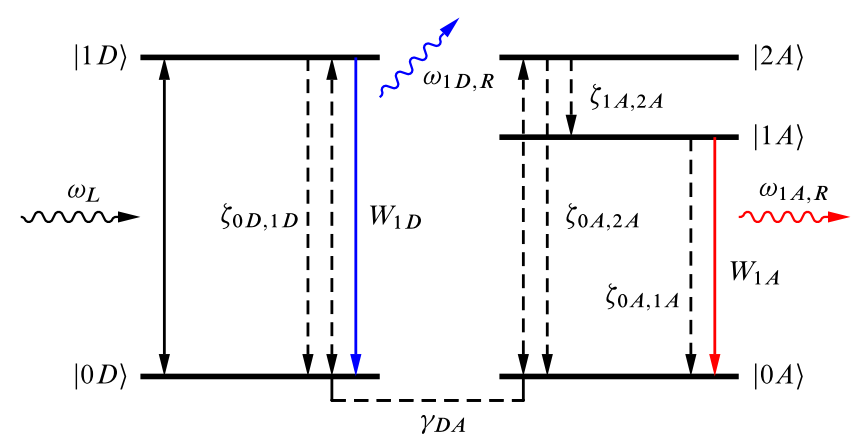

FIG. 4. (Color online) 2-3 scheme of double quantum dot for forbidden optical transitions in QD acceptor. Symbols are the same as in Fig. 3.

states $|1 D\rangle$ and $|2 A\rangle$ are resonant with each other, the incident light can create electron-hole pairs in the $|2 A\rangle$ state of the QD acceptor if this state is allowed for optical transitions (Fig. 3). It is important to keep in mind that the dynamics of the electron-hole pair in the $|2 A\rangle$ state of the QD acceptor will be the same regardless of the excitation method, whether by energy transfer from the QD donor or direct optical generation. The electron-hole pair in the $|2 A\rangle$ state can relax directly to the QD-acceptor ground state $|O A\rangle$ at a rate $\zeta_{0 A, 2 A}$. It can recombine radiatively at a rate $W_{2 A}$, causing emission of photons of frequency $\omega_{2 A, R}$. This pair can recombine nonradiationally with energy transfer at a rate $\gamma_{D A}$ to the QD donor. Finally, the pair can relax to the lowest-energy state $|1 A\rangle$ of the QD acceptor due to intraband transition at a rate $\zeta_{1 A, 2 A}$. This rate is determined by both radiative ${ }^{52}$ and nonradiative ${ }^{12,19,23,26,28}$ intraband transitions. In the final stage of the scenario under consideration, there are two channels of the $|1 A\rangle$-state relaxation. The first channel includes the interband relaxation to the QD-acceptor ground state $|0 A\rangle$ at a rate $\zeta_{0 A, 1 A}$ and the second includes the radiative recombination of the QD-acceptor electron-hole pair at a rate $W_{1 A}$ and emission of photons at a frequency $\omega_{1 A, R}$. Note that, in this case, the total spontaneous secondary emission signal from the double QD will consist of three components, whether or not energy transfer takes place. Indeed, the incident light will directly excite both the QD donor and the QD acceptor. As a result, spontaneous light emission with rates $W_{1 D}, W_{1 A}, W_{2 A}$ and photon frequencies $\omega_{1 D, R}, \omega_{1 A, R}, \omega_{2 A, R}$ will be observed experimentally. The presence of the energytransfer process will only change the relative contributions of these components to the total signal. Another scenario will be realized if the $|2 A\rangle$ state of the QD acceptor is forbidden for optical transitions (Fig. 4). In this case, the QD acceptor can be excited by the energy-transfer process only. As a result, a one-component signal of double QD spontaneous secondary emission from the QD donor will be observed if the energy-transfer rate $\gamma_{D A}$ is negligibly small, e.g., when the distance between QDs is sufficiently large. If the energytransfer rate is high enough, two-component spontaneous light emission with rates $W_{1 D}, W_{1 A}$ and photon frequencies $\omega_{1 D, R}, \omega_{1 A, R}$ from the QD donor and QD acceptor will be observed experimentally. Thus, in contrast with the previous scenario, the presence of spontaneous secondary emission from the QD acceptor is direct evidence of energy transfer between the QD donor and QD acceptor. For the second 


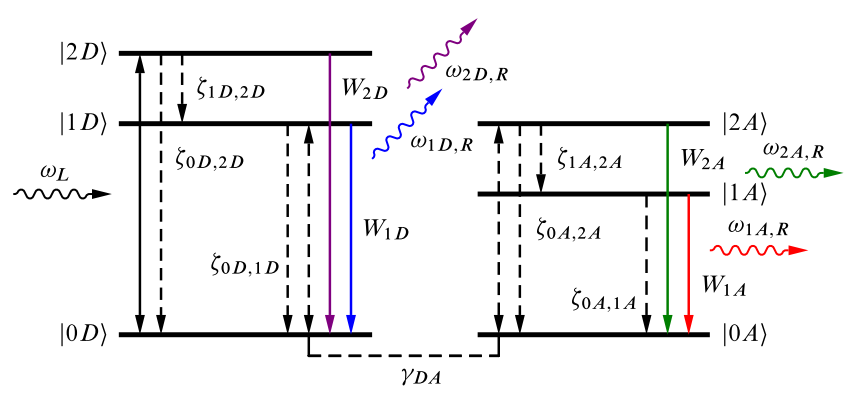

FIG. 5. (Color online) 3-3 scheme of double quantum dot for the allowed optical transitions in the QD acceptor. $\zeta_{0 D, 2 D}$ is the interband relaxation rate for the QD donor. $W_{2 D}$ is the spontaneous light emission rate for the QD donor. $\zeta_{1 D, 2 D}$ is the intraband relaxation rate for the QD donor. $\omega_{2 D, R}$ is the spontaneous light emission frequency. $|2 D\rangle$ is the high-energy state of the QD donor. Other symbols are the same as in Fig. 3.

scenario, the components of the spontaneous light emission can be distinguished easily because their spectral positions $\omega_{1 D, R}$ and $\omega_{1 A, R}$ are quite different.

It is well known (see Refs. 53 and 54) that resonant spontaneous secondary emission consists of resonant scattering and resonant luminescence signals which differ from each other by their magnitudes and spectral widths. This is the case for the $W_{1 D}$ and $W_{2 A}$ contributions to the double QD spontaneous light emission described by the 2-3 scheme (Figs. 3 and 4). Experimentally measured spontaneous secondary emission at the incident light frequency is difficult to analyze and interpret since it consists of the superposition of stray light with $W_{1 D}$ and $W_{2 A}$ components, both determined by scattering and luminescence. These difficulties do not arise for the $W_{1 A}$ contribution to the double QD spontaneous light emission, consisting of the luminescence signal spectrally shifted from the incident light frequency.

Another case of double QD photoexcitation occurs when the incident light, at a frequency $\omega_{L}$, creates electron-hole pairs in the high-energy state $|2 D\rangle$ of the QD donor (Figs. 5 and 6). As before, we consider situations when energy transfer takes place between the lowest-energy state $|1 D\rangle$ of the QD donor and the high-energy state $|2 A\rangle$ of the QD acceptor, which are in resonance each other. In addition, we assume that the incident light cannot excite the QD acceptor directly because of a lack of a suitable electron-hole pair state in the QD acceptor. Evidently, spontaneous light emission in this

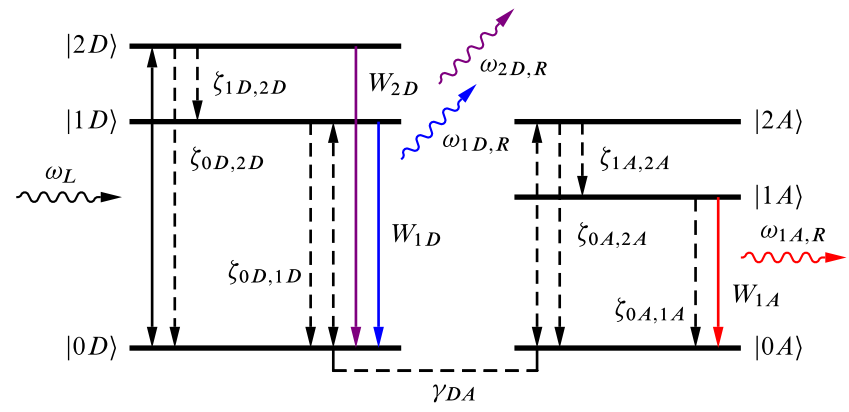

FIG. 6. (Color online) 3-3 scheme of double quantum dot for the forbidden optical transitions in the QD acceptor. Symbols are the same as in Figs. 3 and 5. case may be described using a 3-3 scheme, when three-level models are used for both the QD donor and the QD acceptor. In this process, there are additional steps as compared with the previous case, namely, the intraband relaxation from the high-energy state $|2 D\rangle$ of the QD donor to its lowest-energy state $|1 D\rangle$ at a rate $\zeta_{1 D, 2 D}$, the direct interband relaxation to the QD-donor ground state $|0 D\rangle$ at a rate $\zeta_{0 D, 2 D}$, and the radiative recombination of the $\mathrm{QD}$-donor electron-hole pairs at a rate $W_{2 D}$ and emission of photons of frequency $\omega_{2 D, R}$. In contrast with the first case, the total signal due to double QD spontaneous secondary emission will consist of either three or four components, depending on whether the electron-hole pair state $|2 A\rangle$ of the QD acceptor is forbidden or allowed for optical transitions. In the first scenario, when the $|2 A\rangle$ state is optically allowed (Fig. 5), spontaneous light emission with rates $W_{1 D}, W_{2 D}, W_{1 A}, W_{2 A}$ and photon frequencies $\omega_{1 D, R}$, $\omega_{2 D, R}, \omega_{1 A, R}, \omega_{2 A, R}$ can be observed experimentally. In the second scenario, when the $|2 A\rangle$ state is optically forbidden (Fig. 6), spontaneous light emission at rates $W_{1 D}, W_{2 D}, W_{1 A}$ and photon frequencies $\omega_{1 D, R}, \omega_{2 D, R} \omega_{1 A, R}$ will take place. Obviously, additional contributions to the secondary emission arise due to interband transitions from the resonantly excited high-energy state of the QD donor. It is significant that for both scenarios, QD-acceptor emission of light results from energy transfer from the QD donor to the QD acceptor since the incident light cannot excite the QD acceptor directly. Thus, in contrast with the first case, the presence of spontaneous secondary emission from the QD acceptor is direct evidence of the energy transfer between the QD donor and QD acceptor under both scenarios. When studying the energy-transfer process, the contribution of light emission from the high-energy state $|2 D\rangle$ of the QD donor to the total spontaneous secondary emission signal is of no interest, because its rate, $W_{2 D}$, is independent of the energy-transfer rate $\gamma_{D A}$. Fortunately, it can be easily distinguished from other components of the secondary emission since its spectral position $\omega_{2 D, R}$ differs appreciably from their spectral positions $\omega_{1 D, R}, \omega_{1 A, R}, \omega_{2 A, R}$. We will not discuss this contribution to the total spontaneous secondary emission signal any further here. Note that the residual components of the secondary emission are the luminescent signals spectrally shifted from the incident light frequency.

We require explicit expressions for the population relaxation rates and dephasing rates of the transitions in the QD donor and the QD acceptor in order to explore this further. We assume a simplified relationship between them, summarized by Eq. (22),

$$
\begin{gathered}
\gamma_{1 \alpha, 1 \alpha}^{(0)}=\zeta_{0 \alpha, 1 \alpha}, \\
\gamma_{2 \alpha, 2 \alpha}^{(0)}=\gamma_{1 \alpha, 1 \alpha}^{(0)}+\zeta_{1 \alpha, 2 \alpha}, \\
\bar{\gamma}_{1 \alpha, 0 \alpha}^{(0)}=a_{\alpha} T+b_{\alpha} \bar{n}_{\mathrm{LO}}^{(\alpha)}(T), \\
\gamma_{1 \alpha, 0 \alpha}^{(0)}=\gamma_{1 \alpha, 1 \alpha}^{(0)} / 2+\bar{\gamma}_{1 \alpha, 0 \alpha}^{(0)}, \\
\gamma_{2 \alpha, 0 \alpha}^{(0)}=\gamma_{1 \alpha, 0 \alpha}^{(0)}+\zeta_{1 \alpha, 2 \alpha} / 2,
\end{gathered}
$$

where $a_{\alpha}$ and $b_{\alpha}$ for $\alpha=D, A$ are experimentally obtained constants, $\bar{n}_{\mathrm{LO}}^{(\alpha)}(T)$ is the Bose-Einstein function for 
longitudinal-optical phonons at a frequency $\omega_{\mathrm{LO}}^{(\alpha)}$ and $T$ is the temperature. As mentioned earlier, in our numerical calculations we will consider quantum dots formed from the cubic modification of $\mathrm{CdSe}$ embedded in a $\mathrm{SiO}_{2}$ matrix. In this case $a_{D}=a_{A}=1.5 \times 10^{10} \mathrm{~s}^{-1} \mathrm{~K}^{-1}, \quad b_{D}=b_{A}=2.3 \times 10^{10} \mathrm{~s}^{-1}$, $\hbar \omega_{\mathrm{LO}}^{(D)}=\hbar \omega_{\mathrm{LO}}^{(A)}=26 \mathrm{meV}$, and $\zeta_{0 D, 1 D}=\zeta_{0 A, 1 A}=10^{8} \mathrm{~s}^{-1} \cdot{ }^{29}$

\section{A. Double QD luminescence for the 2-3 scheme}

Consider spontaneous secondary emission from a double QD for the 2-3 scheme of QD electron states (Figs. 3 and 4) which is suitable for the study of both irreversible and reversible incoherent energy transfer. Considering a stationary external excitation $\phi(t)=E_{L}=$ const and using Eqs. (1)-(5), one can obtain the luminescence differential cross section (LDCS) per unit solid angle $\Theta$ and per unit of frequency $\omega_{i \alpha, R}$, where $i=1,2$ and $\alpha=D, A$. Performing a calculation in the lowest order of perturbation theory by interaction with the external classical optical field and with the quantum electromagnetic field of the vacuum, one can obtain the following expressions for the differential cross sections of the donor and acceptor luminescence for incoherent reversible energy transfer for the optically allowed transition $|0 A\rangle$ $\rightarrow|2 A\rangle$ (Fig. 3)

$$
\begin{aligned}
\frac{d^{2} \sigma_{1 D}^{(2-3)}}{d \Theta d \omega_{1 D, R}}= & C\left(\omega_{1 D, R}\right)\left|V_{0 D, 1 D}^{(R)}\right|^{2} L_{D A} \frac{\gamma_{1 D, 0 D}}{\gamma_{1 D, 0 D}^{2}+\Delta_{1 D, R}^{2}} \\
& \times\left\{\left|V_{1 D, 0 D}^{(L)}\right|^{2} \frac{2 \gamma_{2 A, 2 A} \bar{\gamma}_{1 D, 0 D}+\gamma_{D A}^{2}}{2\left(\gamma_{1 D, 0 D}^{2}+\Delta_{1 D, L}^{2}\right)}\right. \\
& \left.+\left|V_{2 A, 0 A}^{(L)}\right|^{2} \frac{\gamma_{D A} \gamma_{2 A, 0 A}}{\gamma_{2 A, 0 A}^{2}+\Delta_{2 A, L}^{2}}\right\},
\end{aligned}
$$

$$
\begin{aligned}
\frac{d^{2} \sigma_{1 A}^{(2-3)}}{d \Theta d \omega_{1 A, R}}= & C\left(\omega_{1 A, R}\right)\left|V_{0 A, 1 A}^{(R)}\right|^{2} \frac{\zeta_{1 A, 2 A} L_{D A}}{\gamma_{1 A, 1 A}^{(0)}} \frac{\gamma_{1 A, 0 A}^{(0)}}{\gamma_{1 A, 0 A}^{(0) 2}+\Delta_{1 A, R}^{2}} \\
& \times\left\{\left|V_{1 D, 0 D}^{(L)}\right|^{2} \frac{\gamma_{D A} \gamma_{1 D, 0 D}}{\gamma_{1 D, 0 D}^{2}+\Delta_{1 D, L}^{2}}\right. \\
& \left.+\left|V_{2 A, 0 A}^{(L)}\right|^{2} \frac{\gamma_{1 D, 1 D} \gamma_{2 A, 0 A}}{\gamma_{2 A, 0 A}^{2}+\Delta_{2 A, L}^{2}}\right\},
\end{aligned}
$$

$$
\begin{aligned}
\frac{d^{2} \sigma_{2 A}^{(2-3)}}{d \Theta d \omega_{2 A, R}}= & C\left(\omega_{2 A, R}\right)\left|V_{0 A, 2 A}^{(R)}\right|^{2} L_{D A} \frac{\gamma_{2 A, 0 A}}{\gamma_{2 A, 0 A}^{2}+\Delta_{2 A, R}^{2}} \\
& \times\left\{\left|V_{1 D, 0 D}^{(L)}\right|^{2} \frac{\gamma_{D A} \gamma_{1 D, 0 D}}{\gamma_{1 D, 0 D}^{2}+\Delta_{1 D, L}^{2}}\right. \\
& \left.+\left|V_{2 A, 0 A}^{(L)}\right|^{2} \frac{2 \gamma_{1 D, 1 D} \bar{\gamma}_{2 A, 0 A}+\gamma_{D A}^{2}}{2\left(\gamma_{2 A, 0 A}^{2}+\Delta_{2 A, L}^{2}\right)}\right\},
\end{aligned}
$$

where $\quad C\left(\omega_{i \alpha, R}\right)=4 \omega_{i \alpha, R}^{4} /\left(\pi c^{4} \hbar^{2}\right), \quad L_{D A}=\left(\gamma_{1 D, 1 D} \gamma_{2 A, 2 A}\right.$ $\left.-\gamma_{D A}^{2}\right)^{-1}, \Delta_{i \alpha, L}=\omega_{i \alpha}-\omega_{L}$, and $\Delta_{i \alpha, R}=\omega_{i \alpha}-\omega_{i \alpha, R}$ are the detunings of the frequency of the exciting and emitted light from the frequency of the electronic transition in the state $i \alpha$. It is easy to see that the first and second terms in the curly brackets of Eqs. (23) and (24) correspond to QD-donor and QDacceptor excitation by the incident light, respectively. As noted above, secondary emission from double QDs contains contributions from the resonant scattering signals. Using our approach, one obtains the following expressions for the differential cross sections of the donor and acceptor resonant scattering

$$
\begin{gathered}
\frac{d^{2} \sigma_{1 D, \mathrm{sc}}^{(2-3)}}{d \Theta d \omega_{1 D, R}}=C\left(\omega_{1 D, R}\right)\left|V_{0 D, 1 D}^{(R)}\right|^{2}\left|V_{1 D, 0 D}^{(L)}\right|^{2} \frac{\gamma_{0} / 2}{\gamma_{0}^{2} / 4+\Delta_{L ; 1 D, R}^{2}} \frac{1}{2\left(\gamma_{1 D, 0 D}^{2}+\Delta_{1 D, L}^{2}\right)}, \\
\frac{d^{2} \sigma_{2 A, \mathrm{sc}}^{(2-3)}}{d \Theta d \omega_{2 A, R}}=C\left(\omega_{2 A, R}\right)\left|V_{0 A, 2 A}^{(R)}\right|^{2}\left|V_{2 A, 0 A}^{(L)}\right|^{2} \frac{\gamma_{0} / 2}{\gamma_{0}^{2} / 4+\Delta_{L ; 2 A, R}^{2}} \frac{1}{2\left(\gamma_{2 A, 0 A}^{2}+\Delta_{2 A, L}^{2}\right)},
\end{gathered}
$$

where $\Delta_{L ; 1 D, R}=\omega_{L}-\omega_{1 D, R}, \Delta_{L ; 2 A, R}=\omega_{L}-\omega_{2 A, R}$, and $\gamma_{0}$ is the spectral width of the initial state. ${ }^{55}$ When the incident light intensity is low, $\gamma_{0}$ is determined by the inverse lifetime of the photons and $\gamma_{0} \ll \gamma_{i \alpha, i \alpha}$. Comparison of Eqs. (23) and (25)-(27) reveals important differences between the luminescence and scattering spectra. If the incident light frequency $\omega_{L}$ is off resonance with corresponding electronic transitions (i.e., $\omega_{L} \neq \omega_{1 D}, \omega_{2 A}$ ), the peak position of the scattering spectrum will coincide with $\omega_{L}$, whereas the peak position of the luminescence spectrum will coincide with $\omega_{1 D}$ or $\omega_{2 D}$. The spectral width of the scattering band is narrower than that of the luminescence band. At the same $\Delta_{i \alpha, L}$, the maximal value of the scattering spectrum peak is far greater than that of the luminescence spectrum peak, and hence the scattering signal can mask the luminescence signal strongly. The luminescence and scattering signals have a different dependence on the energy-transfer rate. Since the scattering signals, Eqs. (26) and (27), depend on $\gamma_{D A}$ only via the resonant denominators $\gamma_{1 D, 0 D}^{2}+\Delta_{1 D, L}^{2}$ and $\gamma_{2 A, 0 A}^{2}+\Delta_{2 A, L}^{2}$, information about the energy-transfer process can be obtained by recording the excitation spectra of resonant scattering. The excitation spectrum is the dependence of the differential cross section, Eq. (26) and (27), on the incident light frequency $\omega_{L}$ at a fixed value of $\Delta_{L ; 1 D, R}$ or $\Delta_{L ; 2 A, R}$. Evidently, the experimental excitation spectrum will consist of two bands with peak positions at $\omega_{L}=\omega_{1 D}$ and $\omega_{L}=\omega_{2 A}$. The full widths of the bands at half maximum are equal to $2 \gamma_{1 D, O D}$ and $2 \gamma_{2 A, 0 A}$, i.e., they depend on $\gamma_{D A}$ directly [see Eqs. (26) and (27)]. Thus, the experi- 
mental data should be analyzed by two Lorentzian fitting. An alternative approach to investigate the energy-transfer process is to examine the dependence of the resonant scattering differential cross sections (RSDCS) on the value of $\gamma_{D A}$, which is varied by changing the interdot distance $r$, or the specimen temperature $T$. When $\omega_{1 D}=\omega_{2 A}=\omega_{L}$, the RSDCS maxima are determined by the following expressions:

$$
\begin{gathered}
\max \frac{d^{2} \sigma_{1 D, \mathrm{sc}}^{(2-3)}}{d \Theta d \omega_{1 D, R}}=\frac{C\left(\omega_{1 D, R}\right)}{\gamma_{0} \gamma_{1 D, 0 D}^{2}}\left|V_{0 D, 1 D}^{(R)}\right|^{2}\left|V_{1 D, 0 D}^{(L)}\right|^{2}, \\
\max \frac{d^{2} \sigma_{2 A, \mathrm{sc}}^{(2-3)}}{d \Theta d \omega_{2 A, R}}=\frac{C\left(\omega_{2 A, R}\right)}{\gamma_{0} \gamma_{2 A, 0 A}^{2}}\left|V_{0 A, 2 A}^{(R)}\right|^{2}\left|V_{2 A, 0 A}^{(L)}\right|^{2},
\end{gathered}
$$

and $\omega_{1 D, R}=\omega_{2 A, R}=\omega_{L}$. Using the function $A / \gamma_{1 D, 0 D}^{2}$ $+B / \gamma_{2 A, 0 A}^{2}$ to fit the experimental data, one can obtain the dependence of $\gamma_{D A}(r, T)$ on the interdot distance or temperature. However, this is more difficult than analyzing the spectral width of the excitation spectra, since it requires either a measurement of the absolute values of the RSDCS to obtain $\gamma_{D A}(r)$, or a knowledge of the explicit temperature dependencies of the QD material and relaxation parameters for finding $\gamma_{D A}(T)$. For resonant luminescence [see Eqs. (23) and (25)], one can obtain information about $\gamma_{D A}$ from the width of the luminescence spectra recorded at fixed values of the incident light frequency $\omega_{L}$, because the LDCSs as compared with the RSDCSs have the additional resonant denominators $\gamma_{1 D, 0 D}^{2}$ $+\Delta_{1 D, R}^{2}$ and $\gamma_{2 A, 0 A}^{2}+\Delta_{2 A, R}^{2}$. In this case, two Lorentzian fitting to the experimental data allows $\gamma_{1 D, 0 D}$ and $\gamma_{2 A, 0 A}$ and hence $\gamma_{D A}$ to be obtained. Of course, one can use the excitation spectra of resonant luminescence in order to obtain $\gamma_{D A}$ in a similar manner to the procedure used for resonant scattering considered above. As before, for resonant scattering, one can investigate the dependence of the LDCS maxima on the value of $\gamma_{D A}(r, T)$,

$$
\begin{aligned}
\max \frac{d^{2} \sigma_{1 D}^{(2-3)}}{d \Theta d \omega_{1 D, R}}= & C\left(\omega_{1 D, R}\right)\left|V_{0 D, 1 D}^{(R)}\right|^{2} \frac{L_{D A}}{\gamma_{1 D, 0 D}} \\
& \times\left\{\left|V_{1 D, 0 D}^{(L)}\right|^{2} \frac{2 \gamma_{2 A, 2 A} \bar{\gamma}_{1 D, 0 D}+\gamma_{D A}^{2}}{2 \gamma_{1 D, 0 D}^{2}}\right. \\
& \left.+\left|V_{2 A, 0 A}^{(L)}\right|^{2} \frac{\gamma_{D A}}{\gamma_{2 A, 0 A}}\right\}, \\
\max \frac{d^{2} \sigma_{2 A}^{(2-3)}}{d \Theta d \omega_{2 A, R}}= & C\left(\omega_{2 A, R}\right)\left|V_{0 A, 2 A}^{(R)}\right|^{2} \frac{L_{D A}}{\gamma_{2 A, 0 A}} \\
& \times\left\{\left|V_{2 A, 0 A}^{(L)}\right|^{2} \frac{2 \gamma_{1 D, 1 D} \bar{\gamma}_{2 A, 0 A}+\gamma_{D A}^{2}}{2 \gamma_{2 A, 0 A}^{2}}\right. \\
& \left.+\left|V_{1 D, 0 D}^{(L)}\right|^{2} \frac{\gamma_{D A}}{\gamma_{1 D, 0 D}}\right\} .
\end{aligned}
$$

Finally, consider the QD-acceptor secondary emission due to the optical transitions $|1 A\rangle \rightarrow|0 A\rangle$ (Fig. 3). This signal is the thermalized luminescence, Eq. (24), since it arises following intraband relaxation in the QD acceptor. Like the RSDCSs, the LDCS for the thermalized luminescence depends on the resonant denominators $\gamma_{1 D, 0 D}^{2}+\Delta_{1 D, L}^{2}$ and $\gamma_{2 A, 0 A}^{2}+\Delta_{2 A, L}^{2}$. Therefore, to obtain information about $\gamma_{D A}$ from the spectral width of the luminescence band, one should record the excitation spectra of the thermalized luminescence. As before, one can study the dependence of the LDCS maxima on the value of $\gamma_{D A}(r, T)$,

$$
\begin{aligned}
\max \frac{d^{2} \sigma_{1 A}^{(2-3)}}{d \Theta d \omega_{1 A, R}}= & C\left(\omega_{1 A, R}\right) \\
& \times\left|V_{0 A, 1 A}^{(R)}\right|^{2} \frac{\zeta_{1 A, 2 A} L_{D A}}{\gamma_{1 A, 1 A}^{(0)} \gamma_{1 A, 0 A}^{(0)}}\left\{\left|V_{1 D, 0 D}^{(L)}\right|^{2} \frac{\gamma_{D A}}{\gamma_{1 D, 0 D}}\right. \\
& \left.+\left|V_{2 A, 0 A}^{(L)}\right|^{2} \frac{\gamma_{1 D, 1 D}}{\gamma_{2 A, 0 A}}\right\} .
\end{aligned}
$$

The luminescence and resonant scattering differential cross sections for the case when the transition $|0 A\rangle \rightarrow|2 A\rangle$ is optically forbidden (Fig. 4) are obtained from Eqs. (23)-(32) if the matrix elements $V_{2 A, 0 A}^{(L)}$ and $V_{0 A, 2 A}^{(R)}$ are equal to zero. In this case, the resonant scattering, Eq. (27), and resonant luminescence, Eq. (25), from the QD acceptor disappears, while the expressions for the LDCS, Eq. (23), for the QD donor and one of the LDCS, Eq. (24), for the QD-acceptor thermalized luminescence are simplified drastically. This allows experimental data to be analyzed using single Lorentzian fitting.

Neglecting second-order terms in the energy-transfer rate $\left(\gamma_{D A}^{2} \rightarrow 0\right)$ and the contribution of reverse transfer to the dephasing and lifetime of the QD-acceptor states $\left(\gamma_{2 A, 0 A}\right.$ $\rightarrow \gamma_{2 A, 0 A}^{(0)}$ and $\left.\gamma_{2 A, 2 A} \rightarrow \gamma_{2 A, 2 A}^{(0)}\right)$, one can obtain the LDCSs for incoherent irreversible energy transfer from Eqs. (23)-(25) (see Ref. 29).

The expressions for the LDCS and RSDCS obtained above, however, cannot be directly compared with experimental data since they were obtained under the assumption of infinite frequency resolution of the photon detection system. For stationary excitation, expressions that allow for the finite frequency resolution of real photon detection systems can be obtained from Eqs. (23)-(27) by convoluting them with a filter frequency function $g_{F}\left(\omega_{F}\right)$. Following Ref. 56 we consider the spectral filter as a Fabry-Perot interferometer, so the filter function has the following form:

$$
g_{F}\left(\omega_{F}\right)=\frac{1}{\pi} \frac{\Gamma_{F} / 2}{\left(\Gamma_{F} / 2\right)^{2}+\omega_{F}^{2}},
$$

where $\Gamma_{F}$ is the spectral bandpass of the filter. The observable values of LDCS and RSDCS are given by the following convolution:

$$
\begin{aligned}
\overline{\operatorname{DCS}_{i \alpha}}\left(\Delta_{i \alpha, L}, \Delta_{i \alpha, F}\right)= & \int_{-\infty}^{+\infty} d \Delta_{i \alpha, R} g\left(\Delta_{i \alpha, F}\right. \\
& \left.-\Delta_{i \alpha, R}\right) \operatorname{DCS}_{i \alpha}\left(\Delta_{i \alpha, L}, \Delta_{i \alpha, R}\right) .
\end{aligned}
$$

Here $\Delta_{i \alpha, F}=\omega_{i \alpha}-\omega_{F}$ is the frequency setting of the filter. Calculation of expressions similar to Eq. (34) in our case is straightforward: the widths of Lorentzians with $\Delta_{i \alpha, R}$ in the denominator should be increased by $\Gamma_{F}$ and all detunings $\Delta_{i \alpha, R}$ should be replaced by $\Delta_{i \alpha, F}$. Note that detuning between the radiation and laser frequency can be expressed as 

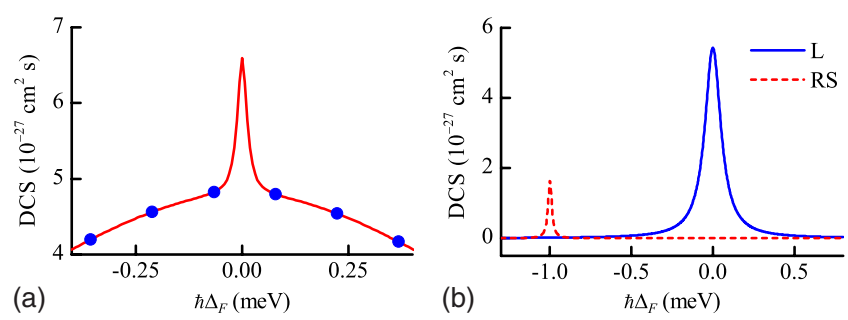

FIG. 7. (Color online) Methods of separation of photoluminescence signal from the secondary emission spectrum of donor QD $\left(T=4 \mathrm{~K}, R_{D}=2 \mathrm{~nm}\right.$, and $\left.\Gamma_{F}=2 \times 10^{10} \mathrm{~s}^{-1}\right)$. (a) Elimination of narrow scattering signal from the wide luminescence contour $\left(\gamma_{i \alpha, 0 \alpha}\right.$ $\gg \Gamma_{F}$ ) with a roughened spectral resolution in case of resonant excitation, $R=3 \mathrm{~nm}$. Solid line depicts the calculated spectrum of donor, Eq. (34), round point positions have been obtained with step of calculation larger than $\Gamma_{F}$. (b) Separation of resonant scattering and luminescence signals by using nonresonant excitation. Here detuning energy $\hbar \Delta_{L}=-1 \mathrm{meV}, R=10 \mathrm{~nm}$, and $\gamma_{0 D, 1 D} \sim \Gamma_{F}$. The luminescence component is defined by the solid and the resonant scattering component by the dashed line.

$\Delta_{L ; i \alpha, R}=\Delta_{i \alpha, R}-\Delta_{i \alpha, L}$, so it should be replaced by $\Delta_{L ; i \alpha, F}$ $=\Delta_{i \alpha, F}-\Delta_{i \alpha, L}$. In most cases, the spectral bandpass of the filter is described by the expression below with dephasing rates $\gamma_{i \alpha, 0 \alpha}$ and a spectral width of the initial state $\gamma_{0}$,

$$
\gamma_{0} / 2 \ll \Gamma_{F} / 2 \ll \gamma_{i \alpha, 0 \alpha},
$$

so we can neglect $\gamma_{0} / 2$ in $\overline{\operatorname{RSDCS}_{i \alpha}}$ and $\Gamma_{F}$ in $\overline{\operatorname{LDCS}_{i \alpha}}$. Thus, consideration of the finite frequency resolution of a FabryPerot interferometer can be done by the following replacements in Eqs. (23)-(27),

$$
\begin{gathered}
\frac{\gamma_{0} / 2}{\left(\gamma_{0} / 2\right)^{2}+\Delta_{L ; i \alpha, R}^{2}} \rightarrow \frac{\Gamma_{F} / 2}{\left(\Gamma_{F} / 2\right)^{2}+\Delta_{L ; i \alpha, F}^{2}}, \\
\frac{\gamma_{i \alpha, 0 \alpha}}{\gamma_{i \alpha, 0 \alpha}^{2}+\Delta_{i \alpha, R}^{2}} \rightarrow \frac{\gamma_{i \alpha, 0 \alpha}}{\gamma_{i \alpha, 0 \alpha}^{2}+\Delta_{i \alpha, F}^{2}} .
\end{gathered}
$$

Separation of the photoluminescence from the total secondary emission signal in experimental spectra can be performed by one of the following procedures. If $\gamma_{i \alpha, 0 \alpha} \gg \Gamma_{F}$, the resonant scattering peak can be excluded by decreasing the spectral resolution. As a result, one can obtain an approximation of a luminescence contour without a sharp peak due to resonant scattering [see Fig. 7(a)]. When the measured widths of the resonant scattering and the luminescence are of the same order, i.e., $\gamma_{i \alpha, 0 \alpha} \sim \Gamma_{F}$, luminescence and resonant scattering can be separated by taking a sufficiently large detuning of the incident photons $\Delta_{L} \gg \Gamma_{F}$. The resonantly scattered photons will have a frequency $\omega_{L}$, whereas the luminescence signal will be observed at $\omega_{i \alpha, R} \neq \omega_{L}$. The spectrum of the donor QD in this case is depicted in Fig. 7(b).

It should be noted that the consideration of reverse energy-transfer processes allows us to avoid the limitation $\gamma_{D A} \ll \gamma_{2 A, 2 A}^{(0)}$ and the validity of our model is limited only by the requirement to have no coherent processes occurring
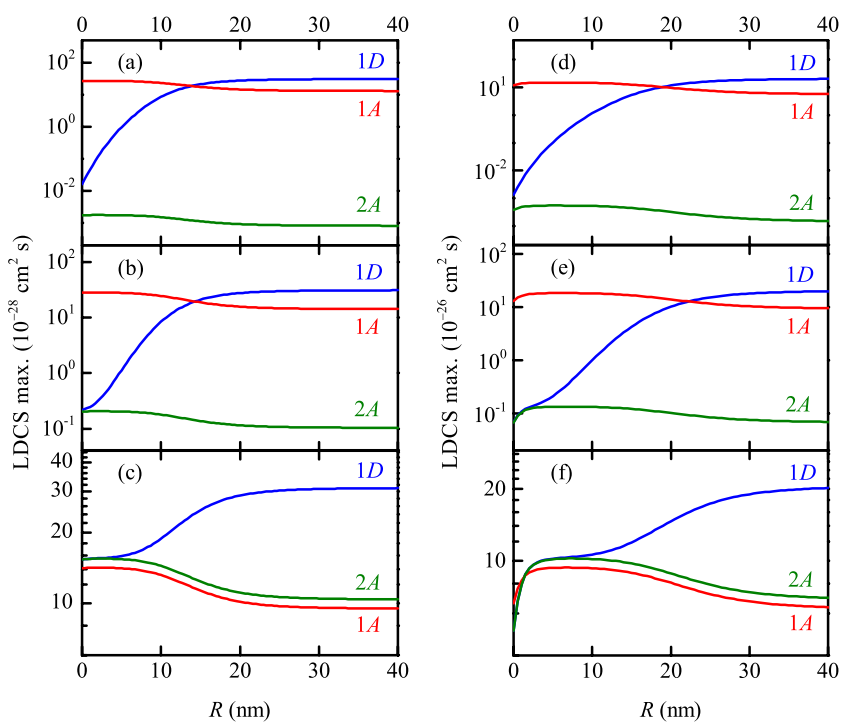

FIG. 8. (Color online) Dependencies of maxima of LDCS on intersurface distance for dipole-allowed energy transfer $\{c 100 ; v 100\} D \rightarrow\{c 110 ; v 110\} A$. The donor relaxation rate is $\gamma_{1 D, 1 D}^{(0)}=10^{8} \mathrm{~s}^{-1}$. (a) $T=300 \mathrm{~K}, \quad \gamma_{2 A, 2 A}^{(0)}=3 \times 10^{12} \mathrm{~s}^{-1}$, (b) $T$ $=300 \mathrm{~K}, \gamma_{2 A, 2 A}^{(0)}=3 \times 10^{10} \mathrm{~s}^{-1}$, (c) $T=300 \mathrm{~K}, \gamma_{2 A, 2 A}^{(0)}=3 \times 10^{8} \mathrm{~s}^{-1}$, (d) $T=90 \mathrm{~K}, \quad \gamma_{2 A, 2 A}^{(0)}=3 \times 10^{12} \mathrm{~s}^{-1}, \quad$ (e) $T=90 \mathrm{~K}, \quad \gamma_{2 A, 2 A}^{(0)}=3$ $\times 10^{10} \mathrm{~s}^{-1}$, and (f) $T=90 \mathrm{~K}, \gamma_{2 A, 2 A}^{(0)}=3 \times 10^{8} \mathrm{~s}^{-1}$.

$$
\Gamma_{D A} \gg \gamma_{D A}
$$

This inequality imposes a limitation on the minimal temperature and interdot radius. In particular, for $\gamma_{1 D, 1 D}^{(0)}=10^{8} \mathrm{~s}^{-1}$, $\gamma_{2 A, 2 A}^{(0)}=3 \times 10^{10} \mathrm{~s}^{-1}, R=1 \mathrm{~nm}, R_{D}=2 \mathrm{~nm}$, for acceptors whose radii satisfy the resonance conditions $\omega_{1 D}\left(R_{D}\right)$
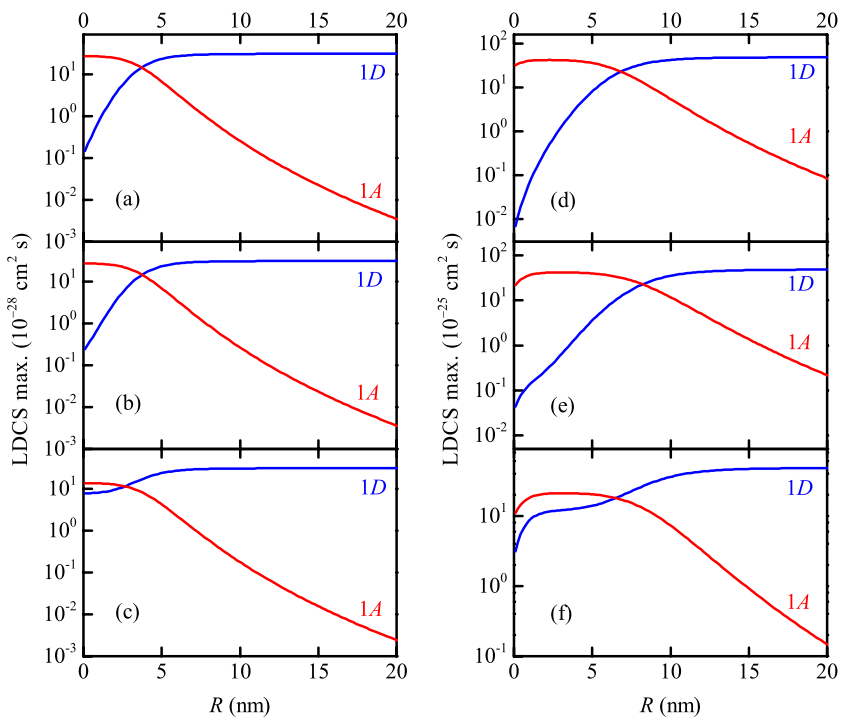

FIG. 9. (Color online) Dependencies of the maxima of the LDCS on intersurface distance for dipole-forbidden energy transfer $\{c 100 ; v 100\} D \rightarrow\{c 100 ; v 110\} A$. The donor relaxation rate is $\gamma_{1 D, 1 D}^{(0)}=10^{8} \mathrm{~s}^{-1}$. (a) $T=300 \mathrm{~K}, \quad \gamma_{2 A, 2 A}^{(0)}=3 \times 10^{12} \mathrm{~s}^{-1}$, (b) $T$ $=300 \mathrm{~K}, \gamma_{2 A, 2 A}^{(0)}=3 \times 10^{10} \mathrm{~s}^{-1}$, (c) $T=300 \mathrm{~K}, \gamma_{2 A, 2 A}^{(0)}=3 \times 10^{8} \mathrm{~s}^{-1}$, (d) $T=30 \mathrm{~K}, \quad \gamma_{2 A, 2 A}^{(0)}=3 \times 10^{12} \mathrm{~s}^{-1}, \quad$ (e) $T=30 \mathrm{~K}, \quad \gamma_{2 A, 2 A}^{(0)}=3$ $\times 10^{10} \mathrm{~s}^{-1}$, and (f) $T=30 \mathrm{~K}, \gamma_{2 A, 2 A}^{(0)}=3 \times 10^{8} \mathrm{~s}^{-1}$. 

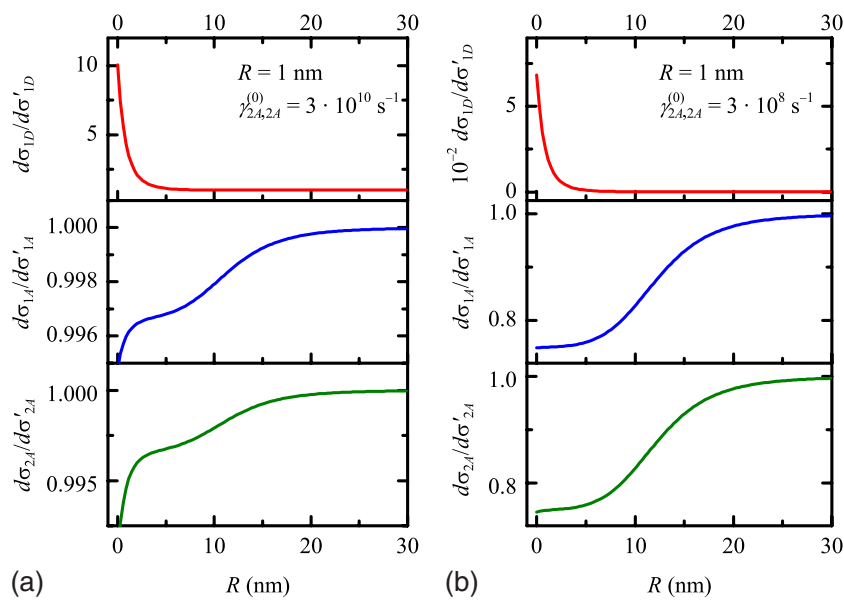

FIG. 10. (Color online) Ratios of LDCSs calculated for the approximation of incoherent reversible $d \sigma_{\beta}$ and irreversible $d \sigma_{\beta}^{\prime}$ energy transport as functions of the intersurface distance at room temperature. (a) $\gamma_{2 A, 2 A}^{(0)}=3 \times 10^{10} \mathrm{~s}^{-1}$ and (b) $\gamma_{2 A, 2 A}^{(0)}=3 \times 10^{8} \mathrm{~s}^{-1}$.

$=\omega_{2 A}\left(R_{A}\right)$, and for strong resonance $\Delta_{D A}=0$, we obtain the following minimal temperature values: $T_{\min }^{(\mathrm{dd})} \gtrsim 53 \mathrm{~K}$ for dipole-dipole transfer $\{c 100 ; v 100\} D \rightarrow\{c 110 ; v 110\} A$, and $T_{\min }^{(\mathrm{dm})} \gtrsim 24 \mathrm{~K}$ for dipole-multipole transfer $\{c 100 ; v 100\} D$ $\rightarrow\{c 100 ; v 110\} A$. When the temperature is relatively low, there is a limitation on the minimal interdot distance that guarantees the absence of coherent processes. For example, for the dipole-dipole energy-transfer process, mentioned earlier, at $T=30 \mathrm{~K}$, the minimal intersurface distance will be $R_{\text {min }}^{(\mathrm{dd})}=2.44 \mathrm{~nm}$.

Let us discuss the dependencies of the luminescence differential cross sections on interdot distance, environmental, and relaxation parameters. Figures 8 and 9 show the dependencies of the maxima of the donor and acceptor LDCS spectra on the distance between the surfaces of the quantum dots for dipole-allowed and dipole-forbidden optical transitions in the QD acceptor at various temperatures. Three different values for the acceptor's relaxation rate are considered. These functions can be divided into three regions. In the first region, where the donor's signal begins to decrease and the acceptor's signals begins to increase, we have incoherent irreversible energy transfer $\left(\gamma_{D A} \ll \gamma_{2 A, 2 A}^{(0)}\right)$. The second region is characterized by the signals at frequencies $\omega_{1 D}$ and $\omega_{2 A}$ becoming equal, so the contribution of the reverse processes is comparable with that of the forward processes $\left(\gamma_{D A} \approx \gamma_{2 A, 2 A}^{(0)}\right)$. In the third region, we observe a rapid decrease in the luminescence signals for all frequencies under consideration. According to Eqs. (23)-(25), this occurs because the energy-transfer rate $\gamma_{D A}$ becomes greater than the pure dephasing rate of the corresponding levels within the quantum dots $\left(\bar{\gamma}_{1 D, 0 D}\right.$ and $\left.\bar{\gamma}_{2 A, 0 A}\right)$, and begins to provide the main contribution to the total dephasing rates $\left(\gamma_{1 D, 0 D}\right.$ and $\left.\gamma_{2 A, 0 A}\right)$. If $\gamma_{D A} \gg \Gamma_{D A}$, the behavior of the system are deter-
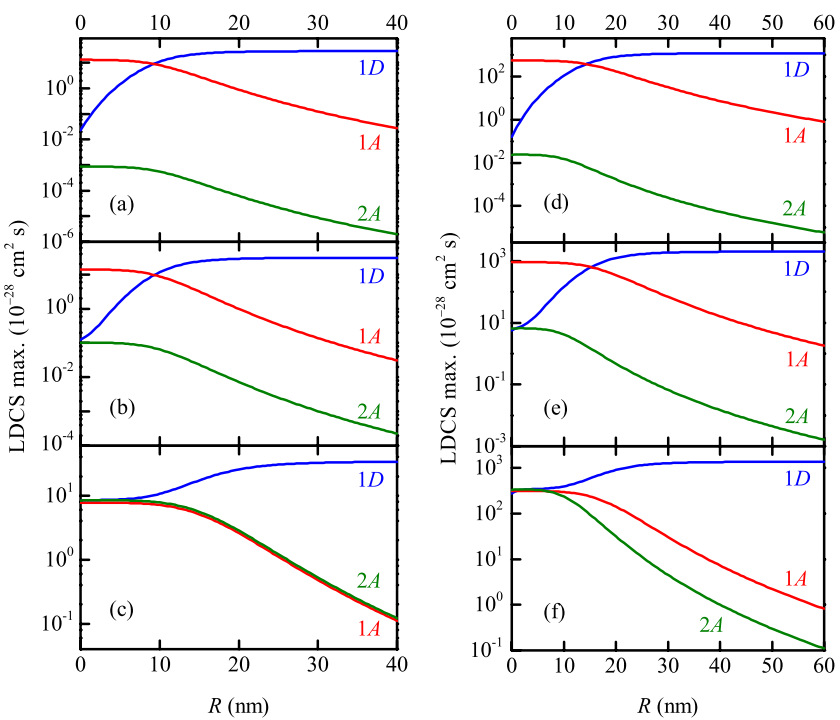

FIG. 11. (Color online) LDCS maximum as a function of intersurface distance for dipole-dipole energy transfer $\{c 100 ; v 100\} D$ $\rightarrow\{c 110 ; v 110\} A$. The external excitation is in resonance with the electron-hole pair $\{c 110 ; v 110\} D$. (a) $T=300 \mathrm{~K}, \gamma_{2 D, 2 D}^{(0)}=\gamma_{2 A, 2 A}^{(0)}=3$ $\times 10^{12} \mathrm{~s}^{-1}$; (b) $T=300 \mathrm{~K}, \quad \gamma_{2 D, 2 D}^{(0)}=\gamma_{2 A, 2 A}^{(0)}=3 \times 10^{10} \mathrm{~s}^{-1}$; (c) $T$ $=300 \mathrm{~K}, \quad \gamma_{2 D, 2 D}^{(0)}=\gamma_{2 A, 2 A}^{(0)}=3 \times 10^{8} \mathrm{~s}^{-1} ; \quad$ (d) $T=90 \mathrm{~K}, \quad \gamma_{2 D, 2 D}^{(0)}$ $=\gamma_{2 A, 2 A}^{(0)}=3 \times 10^{12} \mathrm{~s}^{-1}$; (e) $T=90 \mathrm{~K}, \gamma_{2 D, 2 D}^{(0)}=\gamma_{2 A, 2 A}^{(0)}=3 \times 10^{10} \mathrm{~s}^{-1}$; and (f) $T=90 \mathrm{~K}, \gamma_{2 D, 2 D}^{(0)}=\gamma_{2 A, 2 A}^{(0)}=3 \times 10^{8} \mathrm{~s}^{-1}$.

mined primarily by coherent processes, ${ }^{40}$ and Coulomb interaction leads to entanglement of the donor's and acceptor's electron-hole pairs and removal of their degeneracy. This situation lies beyond the area of validity of our present model, so it is not considered in this study.

The contribution of reverse energy-transfer processes can be estimated by calculating the ratios of the donor and acceptor cross sections using two different approximations: reversible and irreversible energy transfer. Figure 10 depicts the dependencies of these ratios on intersurface distance for two different values of the acceptor's relaxation rate $\gamma_{2 A, 2 A}^{(0)}$. In the first case, when $\gamma_{2 A, 2 A}^{(0)}=3 \times 10^{10} \mathrm{~s}^{-1}$, the acceptor's photoluminescence cross sections do not change significantly due to reverse processes. The situation changes significantly when the acceptor's relaxation rate becomes comparable with that of the donor. From Fig. 10(b), consideration of reverse energy-transfer processes raises the donor's photoluminescence by more than 500 times and lowers the acceptor's signal by $25 \%$.

\section{B. Double quantum dot luminescence for 3-3 scheme}

Let us consider this scheme, when the excited light is not in resonance with the donor transition involved in the nonradiative energy-transfer process (see Figs. 5 and 6). In the case of $|2 A\rangle$ state is dipole allowed (Fig. 5), the LDCS expressions for frequencies shifted from the laser beam have the following form:

$$
\frac{d^{2} \sigma_{1 D}^{(3-3)}}{d \Theta d \omega_{R}}=C\left(\omega_{R}\right)\left|V_{0 D, 1 D}^{(R)}\right|^{2}\left|V_{2 D, 0 D}^{(L)}\right|^{2} \frac{\zeta_{1 D, 2 D} \gamma_{2 A, 2 A} L_{D A}}{\gamma_{2 D, 2 D}^{(0)}} \frac{\gamma_{0 D, 1 D}}{\gamma_{0 D, 1 D}^{2}+\Delta_{1 D, R}^{2}} \frac{\gamma_{2 D, 0 D}^{(0)}+\Delta_{2 D, L}^{(0) 2}}{,},
$$




$$
\begin{gathered}
\frac{d^{2} \sigma_{1 A}^{(3-3)}}{d \Theta d \omega_{R}}=C\left(\omega_{R}\right)\left|V_{0 A, 1 A}^{(R)}\right|^{2}\left|V_{2 D, 0 D}^{(L)}\right|^{2} \frac{\zeta_{1 D, 2 D} \zeta_{1 A, 2 A} \gamma_{D A} L_{D A}}{\gamma_{2 D, 2 D}^{(0)} \gamma_{1 A, 1 A}^{(0)}} \frac{\gamma_{0 A, 1 A}^{(0)}}{\gamma_{0 A, 1 A}^{(0) 2}+\Delta_{1 A, R}^{2}} \frac{\gamma_{2 D, 0 D}^{(0)}}{\gamma_{2 D, 0 D}^{(0) 2}+\Delta_{2 D, L}^{2}}, \\
\frac{d^{2} \sigma_{2 A}^{(3-3)}}{d \Theta d \omega_{R}}=C\left(\omega_{R}\right)\left|V_{0 A, 2 A}^{(R)}\right|^{2}\left|V_{2 D, 0 D}^{(L)}\right|^{2} \frac{\zeta_{1 D, 2 D} \gamma_{D A} L_{D A}}{\gamma_{2 D, 2 D}^{(0)}} \frac{\gamma_{0 A, 2 A}}{\gamma_{0 A, 2 A}^{2}+\Delta_{2 A, R}^{2}} \frac{\gamma_{2 D, 0 D}^{(0)}}{\gamma_{2 D, 0 D}^{(0) 2}+\Delta_{2 D, L}^{2}} .
\end{gathered}
$$

If $|2 A\rangle$ state is dipole forbidden (Fig. 6), the signal, Eq. (37), is absent. These expressions are simpler than Eqs. (23)-(25) for a 2-3 level scheme since the laser radiation excites the donor state only.

Figures 11 and 12 depict the dependencies of the LDCS peak heights on the intersurface distance for different combinations of donor and acceptor relaxation rates. A comparison of Figs. 8 and 9 with Figs. 11 and 12 reveals a major difference between these two schemes. The 3-3 scheme does not exhibit a rapid decrease in the photoluminescence signal for very short interdot distances and for small acceptor relaxation rates. The primary reason for this is that the donor's transition due to light absorption is uncoupled with the energy-transfer transitions (see Figs. 5 and 6) and $\gamma_{D A}$ does not contribute to the dephasing rate of electron-hole pair generation in the donor. Thus, the 3-3 level scheme in closepacked structures of quantum dots allows more effective resonant energy transfer than the 2-3 scheme. Nevertheless, a comparison of LDCS ratios from Fig. 13 shows that reverse energy-transfer processes cause major changes in the photoluminescence signal for low acceptor relaxation rates. In the case of the 3-3 scheme, the increase in the donor signal is
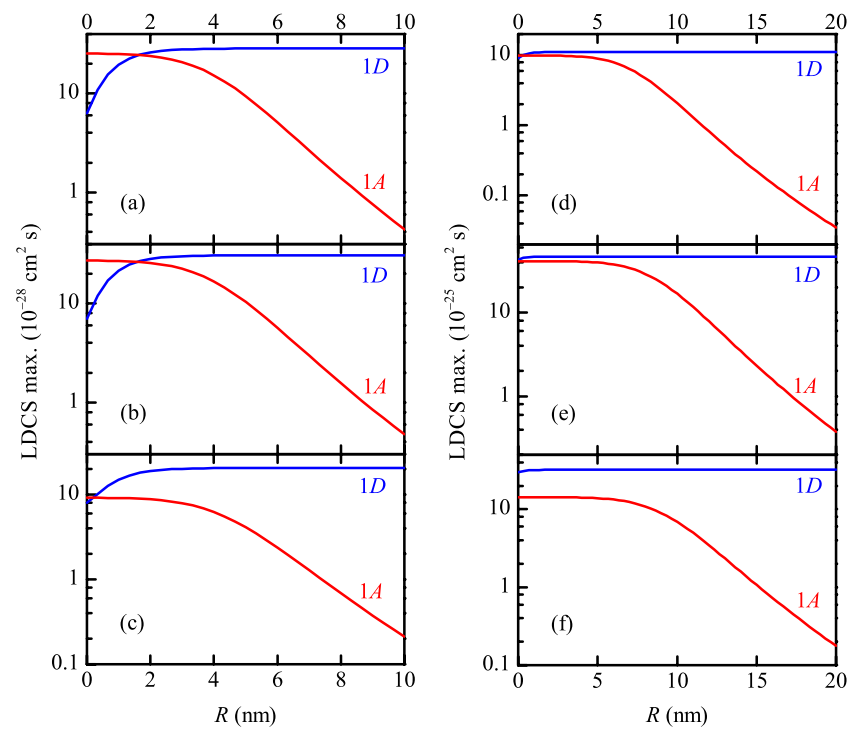

FIG. 12. (Color online) LDCS maximum as a function of intersurface distance for dipole-forbidden energy transfer $\{c 100 ; v 100\} D \rightarrow\{c 100 ; v 110\} A$, scheme $3-3, \gamma_{1 D, 1 D}^{(0)}=10^{8} \mathrm{~s}^{-1}$. (a) $T=300 \mathrm{~K}, \quad \gamma_{2 D, 2 D}^{(0)}=\gamma_{2 A, 2 A}^{(0)}=3 \times 10^{12} \mathrm{~s}^{-1}$; (b) $T=30 \mathrm{~K}, \quad \gamma_{2 D, 2 D}^{(0)}$ $=\gamma_{2 A, 2 A}^{(0)}=3 \times 10^{10} \mathrm{~s}^{-1}$; (c) $T=300 \mathrm{~K}, \gamma_{2 D, 2 D}^{(0)}=\gamma_{2 A, 2 A}^{(0)}=3 \times 10^{8} \mathrm{~s}^{-1}$; (d) $T=30 \mathrm{~K}, \gamma_{2 D, 2 D}^{(0)}=\gamma_{2 A, 2 A}^{(0)}=3 \times 10^{12} \mathrm{~s}^{-1}$; (e) $T=30 \mathrm{~K}, \gamma_{2 D, 2 D}^{(0)}$ $=\gamma_{2 A, 2 A}^{(0)}=3 \times 10^{10} \mathrm{~s}^{-1} ; \quad$ and $\quad(\mathrm{f}) \quad T=30 \mathrm{~K}, \quad \gamma_{2 D, 2 D}^{(0)}=\gamma_{2 A, 2 A}^{(0)}=3$ $\times 10^{8} \mathrm{~s}^{-1}$ nearly two times lower than that for the 2-3 scheme [see Figs. 10(b) and 13(b)].

\section{CONCLUSION}

We have developed a theoretical description of the stationary secondary emission from a double quantum dot, taking into account resonant energy transfer due to Coulomb interaction. Our results allow for the incoherent reversible energy transport that occurs when the intraband relaxation rate in the quantum dot acceptor is comparable to, or less than, the energy-transfer rate. Analytical expressions for the luminescence differential cross section have been obtained for two different level schemes that can be realized experimentally. The first case (2-3 scheme) occurs when the external optical excitation is in resonance with the QD-donor transition involved in the energy-transfer process. The second case (3-3 scheme) occurs when the excitation creates electron-hole pairs in highly energetic donor states which does not take part in energy transfer.

We performed an analysis of the luminescence differential cross-section dependencies on the dimensional and relaxation parameters of the double quantum dot system: viz., interdot distance, dephasing, and relaxation rates of the donor and acceptor. It has been shown that incoherent reversible energy-transfer processes can have a large impact on the optical properties of QD systems with a close-packed
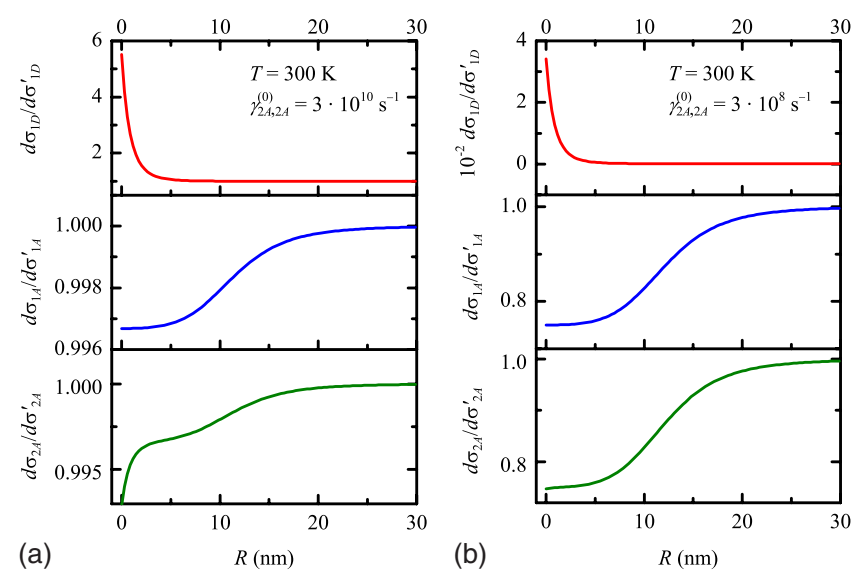

FIG. 13. (Color online) Ratios of LDCSs calculated for the approximation of incoherent reversible $d \sigma_{\beta}$ and irreversible $d \sigma_{\beta}^{\prime}$ energy transport as functions of the intersurface distance at room temperature. (a) $\gamma_{2 D, 2 D}^{(0)}=\gamma_{2 A, 2 A}^{(0)}=3 \times 10^{10} \mathrm{~s}^{-1}$ and (b) $\gamma_{2 D, 2 D}^{(0)}=\gamma_{2 A, 2 A}^{(0)}$ $=3 \times 10^{8} \mathrm{~s}^{-1}$ 
arrangement. Comparison of the luminescence differential cross-section dependencies for different level schemes allows to conclude that the 3-3 scheme results in a better efficiency of resonant energy transfer than the 2-3 scheme when reversible processes are significant, e.g., in the cases of low temperatures, high intraband relaxation rates in acceptor and low interdot distances.

\section{ACKNOWLEDGMENTS}

Three of the authors (S.Yu.K., A.V.F., and A.V.B.) are grateful to the RFBR (Grants No. 09-02-00333 and No. 0902-01439) and the Ministry of Education and Science of the Russian Federation (Grants No. 2.1.1/1933 and No. 2.1.1/ 1880) for partial financial support of this work. *stanislav.kruchinin@gmail.com

†a_v_fedorov@inbox.ru

${ }^{1}$ E. Rozbicki and P. Machnikowski, Phys. Rev. Lett. 100, 027401 (2008).

${ }^{2}$ Z. Tang, N. A. Kotov, and M. Giersig, Science 297, 237 (2002).

${ }^{3}$ S.-K. Hong, S. W. Nam, and K.-H. Yeon, Nanotechnology 19, 155402 (2008).

${ }^{4}$ Z. Tang, Z. Zhang, Y. Wang, S. C. Glotzer, and N. A. Kotov, Science 314, 274 (2006).

${ }^{5}$ A. N. Al-Ahmadi and S. E. Ulloa, Appl. Phys. Lett. 88, 043110 (2006).

${ }^{6}$ A. Sukhanova, A. V. Baranov, T. S. Perova, J. H. M. Cohen, and I. Nabiev, Angew. Chem., Int. Ed. 45, 2048 (2006).

${ }^{7}$ A. Sukhanova, Y. Volkov, A. L. Rogach, A. V. Baranov, A. S. Susha, D. Klinov, V. Oleinikov, J. H. M. Cohen, and I. Nabiev, Nanotechnology 18, 185602 (2007).

${ }^{8}$ V. M. Agranovich and M. D. Galanin, Electronic Excitation Energy Transfer in Condensed Matter (North-Holland, Amsterdam, 1982).

${ }^{9}$ R. D. Schaller, J. M. Pietryga, S. V. Goupalov, M. A. Petruska, S. A. Ivanov, and V. I. Klimov, Phys. Rev. Lett. 95, 196401 (2005).

${ }^{10}$ E. Hendry, M. Koeberg, F. Wang, H. Zhang, C. de Mello Donegá, D. Vanmaekelbergh, and M. Bonn, Phys. Rev. Lett. 96, 057408 (2006).

${ }^{11}$ C. Bonati, A. Cannizzo, D. Tonti, A. Tortschanoff, F. van Mourik, and M. Chergui, Phys. Rev. B 76, 033304 (2007).

${ }^{12}$ P. Guyot-Sionnest, B. Wehrenberg, and D. Yu, J. Chem. Phys. 123, 074709 (2005).

${ }^{13}$ A. Pandey and P. Guyot-Sioneest, Science 322, 929 (2008).

${ }^{14}$ K. Ikeda, H. Sekiguchi, F. Minami, J. Yoshino, Y. Mitsumori, H. Amanai, S. Nagao, and S. Sakaki, J. Lumin. 108, 273 (2004).

${ }^{15}$ R. Heitz, H. Born, F. Guffarth, O. Stier, A. Schliwa, A. Hoffmann, and D. Bimberg, Phys. Rev. B 64, 241305(R) (2001).

${ }^{16}$ U. Bockelmann and G. Bastard, Phys. Rev. B 42, 8947 (1990).

${ }^{17}$ H. Benisty, Phys. Rev. B 51, 13281 (1995).

${ }^{18}$ S. Sanguinetti, M. Guzzi, E. Grilli, M. Gurioli, L. Seravalli, P. Frigeri, S. Franchi, M. Capizzi, S. Mazzuccato, and A. Polimeni, Phys. Rev. B 78, 085313 (2008).

${ }^{19}$ P. C. Sercel, Phys. Rev. B 51, 14532 (1995).

${ }^{20}$ D. F. Schroeter, D. J. Griffiths, and P. C. Sercel, Phys. Rev. B 54, 1486 (1996).

${ }^{21}$ A. N. Poddubny and S. V. Goupalov, Phys. Rev. B 77, 075315 (2008).

${ }^{22}$ U. Bockelmann and T. Egeler, Phys. Rev. B 46, 15574 (1992).

${ }^{23}$ A. L. Efros, V. A. Kharchenko, and M. Rosen, Solid State Commun. 93, 281 (1995).

${ }^{24}$ V. A. Kharchenko and M. Rosen, J. Lumin. 70, 158 (1996).
${ }^{25}$ A. V. Baranov, A. V. Fedorov, I. D. Rukhlenko, and Y. Masumoto, Phys. Rev. B 68, 205318 (2003).

${ }^{26}$ A. V. Fedorov, A. V. Baranov, I. D. Rukhlenko, and S. V. Gaponenko, Phys. Rev. B 71, 195310 (2005).

${ }^{27}$ A. V. Fedorov, A. V. Baranov, I. D. Rukhlenko, T. S. Perova, and K. Berwick, Phys. Rev. B 76, 045332 (2007).

${ }^{28}$ V. I. Klimov, A. A. Mikhailovsky, D. W. McBranch, C. A. Leatherdale, and M. G. Bawendi, Phys. Rev. B 61, R13349 (2000).

${ }^{29}$ S. Yu. Kruchinin, A. V. Fedorov, A. V. Baranov, T. S. Perova, and K. Berwick, Phys. Rev. B 78, 125311 (2008).

${ }^{30}$ C. R. Kagan, C. B. Murray, and M. G. Bawendi, Phys. Rev. B 54, 8633 (1996).

${ }^{31}$ R. Heitz, I. Mukhametzhanov, P. Chen, and A. Madhukar, Phys. Rev. B 58, R10151 (1998).

${ }^{32}$ O. I. Mićić, K. M. Jones, A. Cahill, and A. J. Nozik, J. Phys. Chem. B 102, 9791 (1998).

${ }^{33}$ L. Xu, J. Xu, Z. Ma, W. Li, X. Huang, and K. Chen, Appl. Phys. Lett. 89, 033121 (2006).

${ }^{34}$ K. Nishibayashi, T. Kawazoe, M. Ohtsu, K. Akahane, and N. Yamamoto, Appl. Phys. Lett. 93, 042101 (2008).

${ }^{35}$ T. Franzl, D. S. Koktysh, T. A. Klar, A. L. Rogach, J. Feldmann, and N. Gaponik, Appl. Phys. Lett. 84, 2904 (2004).

${ }^{36}$ P. Bajracharya, T. A. Nguyen, S. Mackowski, L. M. Smith, H. P. Wagner, U. W. Pohl, D. Bimberg, and M. Strassburg, Phys. Rev. B 75, 035321 (2007).

${ }^{37}$ V. Rinnerbauer et al., Phys. Rev. B 77, 085322 (2008).

${ }^{38}$ K. Hosoki, T. Tayagaki, S. Yamamoto, K. Matsuda, and Y. Kanemitsu, Phys. Rev. Lett. 100, 207404 (2008).

${ }^{39}$ G. D. Scholes and D. L. Andrews, Phys. Rev. B 72, 125331 (2005).

${ }^{40}$ A. Nazir, B. W. Lovett, S. D. Barrett, J. H. Reina, and G. A. D. Briggs, Phys. Rev. B 71, 045334 (2005).

${ }^{41}$ G. Allan and C. Delerue, Phys. Rev. B 75, 195311 (2007).

${ }^{42}$ A. O. Govorov, Phys. Rev. B 71, 155323 (2005).

${ }^{43}$ K. Blum, Density Matrix Theory And Applications (Plenum Press, New York, 1981).

${ }^{44}$ U. Fano, Lectures on the Many-Body Problem (Academic Press, New York, 1964), Vol. 2.

${ }^{45}$ C. de Mello Donegá, P. Liljeroth, and D. Vanmaekelbergh, Small 1, 1152 (2005).

${ }^{46}$ A. V. Baranov, K. Inoue, K. Toba, A. Yamanaka, V. I. Petrov, and A. V. Fedorov, Phys. Rev. B 53, R1721 (1996).

${ }^{47}$ K. Inoue, A. Yamanaka, K. Toba, A. V. Baranov, A. A. Onushchenko, and A. V. Fedorov, Phys. Rev. B 54, R8321 (1996).

${ }^{48}$ R. Wargnier, A. V. Baranov, V. G. Maslov, V. Stsiapura, M. Artemyev, M. Pluot, A. Sukhanova, and I. Nabiev, Nano Lett. 4, 451 (2004). 
${ }^{49}$ A. I. Anselm, Introduction to Semiconductor Theory (PrenticeHall, Englewood Cliffs, NJ, 1978).

${ }^{50}$ D. J. Norris and M. G. Bawendi, Phys. Rev. B 53, 16338 (1996).

${ }^{51}$ L. M. Landsberger and W. A. Tiller, Appl. Phys. Lett. 49, 143 (1986).

${ }^{52}$ A. Germeau, A. L. Roest, D. Vanmaekelbergh, G. Allan, C. Delerue, and E. A. Meulenkamp, Phys. Rev. Lett. 90, 097401 (2003).
${ }^{53}$ Y. R. Shen, Phys. Rev. B 9, 622 (1974).

${ }^{54}$ A. V. Fedorov, A. V. Baranov, and Y. Masumoto, Opt. Spectrosc. 93, 555 (2002).

${ }^{55} \mathrm{~W}$. Heitler, The Quantum Theory of Radiation (Clarendon Press, Oxford, 1954).

${ }^{56}$ J. S. Melinger and A. C. Albrecht, J. Chem. Phys. 84, 1247 (1986). 